\title{
Deconstructing Lawvere with Distributive Laws
}

\author{
Filippo Bonchi ${ }^{\mathrm{a}}$, Paweł Sobociński ${ }^{\mathrm{b}}$, Fabio Zanasi ${ }^{\mathrm{c}}$ \\ ${ }^{a}$ Università di Pisa, Italy \\ ${ }^{b}$ University of Southampton, United Kingdom \\ ${ }^{c}$ University College London, United Kingdom
}

\begin{abstract}
PROs, PROPs and Lawvere categories are related notions adapted to the study of algebraic structures borne by an object in a category: PROs are monoidal, PROPs are symmetric monoidal and Lawvere categories are cartesian. This paper connects the three notions using Lack's technique for composing $\mathrm{PRO}(\mathrm{P}) \mathrm{s}$ via distributive laws. We show that Lawvere categories can be seen as the composite PROP $\mathrm{CCm} ; \mathcal{T}$, where $\mathcal{T}$ expresses the algebraic structure in linear form and $\mathrm{CCm}$ express the ability of copying and discarding them. In turn the PROP $\mathcal{T}$ can be decomposed in terms of PROs as $\mathrm{P} ; \mathrm{S}$ where $\mathrm{P}$ expresses the ability of permuting variables and $\mathrm{S}$ is the PRO encoding the syntactic structure without permutations.
\end{abstract}

\section{Introduction}

The subject of this paper is presentations of algebraic theories, defined as the pair $(\Sigma, E)$ given by a signature $\Sigma$ - a set of operations with associated arity and coarity - and a set $E$ of equations between $\Sigma$-terms. Presentations play a central role in theoretical computer science, serving as specifications for various computational models. For instance, a presentation captures the core of a programming language, with $\Sigma$-terms defining the language syntax and $E$ describing a notion of semantic equality between programs. In such classical examples it is typical to deal with cartesian presentations, in which $\Sigma$-operations all have coarity 1 and $\Sigma$-terms are constructed as trees with nodes labeled by operations in $\Sigma$, or variables. In recent years, much

Email addresses: filippo.bonchi@unipi.it (Filippo Bonchi), ps@ecs.soton.ac.uk (Paweł Sobociński), f.zanasi@ucl.ac.uk (Fabio Zanasi) 
attention has been focussed on weakening the "cartesianity" of algebraic theories, which in essence amounts to resource sensitivity: dropping the assumption that the underlying data manipulated by an algebraic theory is classical. Symmetric monoidal algebraic theories have been of particular interest, which leads us to consider symmetric monoidal presentations (SMPs). SMPs are a natural presentation of diagrammatic formalisms, including those used for circuits and other component-based systems, which are sensitive to resources. SMPs feature in algebraic approaches to Petri nets [16, 41], bigraphs [17], quantum circuits [20], and signal flow graphs [7, 9, 1, 25, 11]. A crucial difference to cartesian presentations is linearity: variables of symmetric monoidal terms cannot be copied nor discarded. Moreover, whereas in cartesian presentations all operations have coarity 1, signatures of SMPs may feature operations with (arbitrary arities) and coarities. The resulting terms are 2-dimensional: the structure is that of directed acyclic graphs, rather than trees.

A further generalisation, relevant in mathematical physics (see e.g. [2]), is provided by monoidal presentations (MPs) of merely monoidal algebraic theories. As in symmetric monoidal presentations, the use of variables is linear, but variables cannot be permuted: in every pair of compatible $\Sigma$ terms they must be used in the same order.

In this paper we develop a stratified treatment of monoidal, symmetric monoidal and cartesian presentations. Roughly speaking, a symmetric monoidal presentation can obtained from a monoidal presentation by letting it interact with a permutation structure; in turn, a cartesian presentation arises from the interaction of a symmetric monoidal presentation with a structure for copying and discarding variables. Our technical contribution is to exhibit these constructions in a unified and canonical manner using distributive laws of PROs and PROPs [34].

PROs and PROPs are, respectively, monoidal and symmetric monoidal categories with objects the natural numbers, where monoidal product on objects is addition. They are the obvious choices for the notion of category induced by, respectively, monoidal and symmetric monoidal presentations. Indeed, monoidal presentations freely generate PROs and symmetric monoidal presentations generate PROPs. Both PROs and PROPs can be regarded as monads in the 2-categorical sense of Street [42] and, like monads, they can be composed by means of distributive laws 31. In a nutshell, a distributive law $\lambda: \mathcal{T}_{1} ; \mathcal{T}_{2} \rightarrow \mathcal{T}_{2} ; \mathcal{T}_{1}$ of $\mathrm{PRO}(\mathrm{P}) \mathrm{s}$ is a recipe for moving arrows of $\mathcal{T}_{1}$ past those 
of $\mathcal{T}_{2}$. The resulting $\mathrm{PRO}(\mathrm{P}) \mathcal{T}_{2} ; \mathcal{T}_{1}$ enjoys a factorisation property: every arrow in $\mathcal{T}_{2} ; \mathcal{T}_{1}$ decomposes as one of $\mathcal{T}_{2}$ followed by one of $\mathcal{T}_{1}$. The graph of $\lambda$ can be seen as a set of directed equations $E_{\lambda}:=\left(\stackrel{\in \mathcal{T}_{1}}{\longrightarrow} \stackrel{\in \mathcal{T}_{2}}{\longrightarrow}\right) \approx\left(\stackrel{\in \mathcal{T}_{2}}{\longrightarrow} \stackrel{\in \mathcal{T}_{1}}{\longrightarrow}\right)$, which also can be used as an "oracle" to obtain a presentation of $\mathcal{T}_{2} ; \mathcal{T}_{1}$ : if $\mathcal{T}_{1}$ and $\mathcal{T}_{2}$ are presented by $\left(\Sigma_{1}, E_{1}\right)$ and $\left(\Sigma_{2}, E_{2}\right), \mathcal{T}_{2} ; \mathcal{T}_{1}$ is presented by $\left(\Sigma_{1} \uplus \Sigma_{2}, E_{1} \uplus E_{2} \uplus E_{\lambda}\right)$.

The cartesian analogues of PROs and PROPs are Lawvere categories 1 , a well known class of structures adapted to the study of categorical universal algebra. Our starting observation is that the Lawvere category $\mathcal{L}_{\Sigma}$ on a cartesian signature $\Sigma$ exhibits a factorisation property analogous to the one of composed PROPs: arrows can be decomposed as $\stackrel{\in \mathrm{CCm}}{\longrightarrow} \stackrel{\in \mathcal{T}_{\Sigma}}{\longrightarrow}$, where $\mathrm{CCm}$ is the PROP of commutative comonoids, generated by a copy $1 \rightarrow 2$ and a discard $1 \rightarrow 0$ operation together with the usual equations, and $\mathcal{T}_{\Sigma}$ is the PROP freely generated by $\Sigma$. This factorisation represents cartesian $\Sigma$-terms by their syntactic tree - the $\mathcal{T}_{\Sigma}$-part — with the possibility of explicitly indicating variable-sharing among sub-terms - the CCm-part. This simple observation leads us to the main result of the paper: for a cartesian signature $\Sigma$, there is a distributive law of PROPs $\lambda: \mathcal{T}_{\Sigma} ; \mathrm{CCm} \rightarrow \mathrm{CCm} ; \mathcal{T}_{\Sigma}$ which is presented by equations that express the naturality of copier and discarder; the resulting composed PROP $\mathrm{CCm} ; \mathcal{T}_{\Sigma}$ is the Lawvere category $\mathcal{L}_{\Sigma}$.

To prove this, another observation is useful: the PROP $\mathcal{T}_{\Sigma}$ itself enjoys the factorisation property of composed PROs: arrows can be decomposed as $\stackrel{\in \mathrm{P}}{\longrightarrow} \stackrel{\in \mathrm{S}_{\Sigma}}{\longrightarrow}$, where $\mathrm{S}_{\Sigma}$ is the PRO freely generated by $\Sigma$ and $\mathrm{P}$ is the PRO of permutations. From this, we deduce the existence of a distributive law of PROs $\lambda^{\prime}: \mathrm{S}_{\Sigma} ; \mathrm{P} \rightarrow \mathrm{P} ; \mathrm{S}_{\Sigma}$ which is presented by equations telling that permutations are well-behaved with respect to $\Sigma$.

A quotient construction on the distributive laws $\lambda$ and $\lambda^{\prime}$ leads to a more general observation about cartesian presentations $(\Sigma, E)$, with the proviso that $E$ only contains linear equations. For instance the Lawvere category of commutative monoids $\mathcal{L}_{\mathrm{CMn}}$ can be obtained directly by means of PROP composition, while the Lawvere category $\mathcal{L}_{\text {Gr }}$ of abelian groups cannot, because of the presence of the non-linear equation $x \times x^{-1}=1$ for the inverse of the group action. Obviously, one can still formulate $\mathcal{L}_{\mathrm{Gr}}$ as the quotient of the composite $\mathcal{L}_{\mathrm{CMn}}$ by adding this equation (see Example 6.2(b)). Similarly, the

${ }^{1}$ Also called Lawvere theories in the literature: i.e. finite product categories with set of objects the natural numbers, where product on objects is addition [33, 29]. 
PROP of monoids Mn can be obtained by means of PRO composition, but the PROP of commutative monoids cannot, since the axioms expressing commutativity $x \times y=y \times x$ relies on the "availability" of permutations. Again, this can be regarded as a quotient of the composite Mn (see Example 3.3(b)].

Related work. In categorical algebra, following Lawvere's influential perspective [33], presentations are sometimes deemed as a derivative concept, since unlike theories (Lawvere categories) they are not invariant in regards to models: different presentations can yield the same theory, i.e. the same Lawvere category. In our work, instead, presentations take on a more central role. As explained above, for us this is motivated by the ubiquitous role that presentations play in theoretical computer science as specifications for componentbased systems. This paper brings a more structured approach to the design of such specifications, modularising notoriously difficult tasks such as obtaining completeness and decidability of a string diagrammatic theory with respect to an intended semantics.

The motto "cartesian terms = linear terms + copying and discarding" inspired several papers exploiting the role of $\mathrm{CCm}$ in Lawvere categories, see e.g. [18, 32, 21]. In our work, Lawvere categories feature as a distinguished example of a construction, PROP composition, that is increasingly important in many recent research threads [8, 7, 25, 38]. It is also worth mentioning that the relationship between symmetric monoidal and cartesian structures is central in the categorical semantics of linear logic; from this perspective, the presence of $\mathrm{CCm}$ allows the interpretation of the structural rules of contraction and weakening — see e.g. [30, 36].

The following construction in two-dimensional monad theory [5], reported by Baez in [3], is close in spirit to our work. There is pseudo-adjunction between symmetric monoidal and categories with finite products

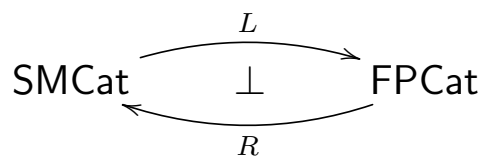

where $R$ is the forgetful functor and $L$ adds to any object of $\mathbb{C} \in$ SMCat a natural copy-discard structure: natural diagonals and projections. Baez [3] describes an equivalence between $R L(\mathbb{C})$ and $\mathbb{C} \otimes \mathrm{CCm}$, with the tensor product $\otimes$ defined by SMCat $\left[\mathbb{C}_{1} \otimes \mathbb{C}_{2}, \mathbb{C}_{3}\right] \simeq \operatorname{SMCat}\left[\mathbb{C}_{1}, \operatorname{SMCat}\left[\mathbb{C}_{2}, \mathbb{C}_{3}\right]\right]$.

In this paper, we restrict our attention to PROPs $\mathcal{T}_{\Sigma} \in$ SMCat freely generated by a cartesian signature $\Sigma$ : here it is enough to add a copy-discard 
structure for the object 1 (producing only finitely many additional equations) and $R L\left(\mathcal{T}_{\Sigma}\right)$ coincides with PROP composition $\mathrm{CCm} ; \mathcal{T}_{\Sigma}$. Indeed, combining $\mathcal{T}_{\Sigma}$ and $\mathrm{CCm}$ by tensor or by composition (according to the distributive law of Theorem 6.1 below) produces the same equations (Lw1)-(Lw2) $]^{2}$. In this particular setting, therefore, the tensor product and the distributive law give the same result.

The two constructions - tensor products and distributive laws - differ for permutations: the $\mathrm{PROP} \mathcal{T}_{\Sigma}$ is the composite $\mathrm{PRO} \mathrm{P} ; \mathrm{S}_{\Sigma}$ (Theorem 5.1) but does not result from a tensor of $\mathrm{P}$ and $\mathrm{S}_{\Sigma}$. Indeed, the aforementioned distributive law gives equations (N1)-( $(\mathrm{N2})$, whereas the tensor means that S-operations and symmetries must "commute" [28, 27]. This is a weaker condition than naturality: $\Sigma$-operations can only "slide" over a symmetry in the restricted case illustrated below:

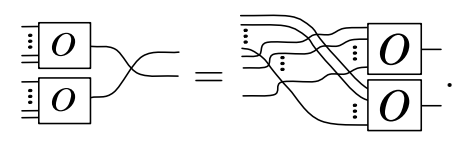

Another example where the two constructions diverge is the well-known symmetric monoidal theory of special (strongly separable) Frobenius algebgras. As shown by Lack [31], this important theory arises from a distributive law of commutative monoids over commutative comonoids, from which one can read off the usual presentation. The composite here is the PROP of cospans of finite sets. The tensor product, instead, gives the theory of bialgebras (see e.g. [3]), which incidentally can be obtained from a different distributive law, that of comonoids over monoids, forming the composite PROP of spans of finite sets [31. It is this finer control and increased expressivity of distributive laws as a powerful source of "equation provenance" that makes it interesting for us as a tool for the modular construction of algebraic theories.

We conclude with an observation that relates our work to earlier studies of syntax with non-linear use of variables. Another operation on PROPs, namely sum, is often useful when working with symmetric monoidal presentations [43]. Sum is just the coproduct in the category of PROPs. Whenever two PROPs $\mathcal{T}_{1}$ and $\mathcal{T}_{2}$ can be presented by the presentations $\left(\Sigma_{1}, E_{1}\right)$ and $\left(\Sigma_{2}, E_{2}\right)$, then their sum $\mathcal{T}_{1}+\mathcal{T}_{2}$ is presented by the disjoint union $\left(\Sigma_{1} \uplus \Sigma_{2}, E_{1} \uplus E_{2}\right)$.

\footnotetext{
${ }^{2}$ For computing the equations presenting the tensor of theories, see e.g. [27, Sec. 4].
} 
By taking the sum $\mathrm{CCm}+\mathcal{T}_{\Sigma}$, rather than the composition, we are able to capture a different, well-known representation for cartesian $\Sigma$-terms, namely term graphs, which are acyclic graphs labeled over $\Sigma$. With respect to the standard tree representation, the benefit of term graphs is that the sharing of any common sub-term can be represented explicitly, making them particularly appealing for efficient rewriting algorithms, see e.g. 40. for a survey on the subject. As shown in [21], $\Sigma$-term graphs are in 1-1 correspondence with the arrows of the free gs-monoidal category generated by $\Sigma$, a concept that actually amounts to forming the sum of PROPs $\mathrm{CCm}+\mathcal{T}_{\Sigma}$. Thus the only difference between term graphs and the representation of terms given by $\mathrm{CCm} ; \mathcal{T}_{\Sigma}$ is in the naturality of copier and discarder in the latter. For instance, a term where a resource is explicitly copied is not identified with the term where two copies appear separately: i.e., copying is not natural.

Previous work. This work extends the conference paper [10] with material on PROs and distributive laws of PROs. As well as describing a more comprehensive picture - monoidal presentations $\rightsquigarrow$ symmetric monoidal presentations $\rightsquigarrow$ cartesian presentations - the new decomposition of $\mathcal{T}_{\Sigma}$ as $\mathrm{P} ; \mathrm{S}_{\Sigma}$ (Theorem 5.1) simplifies the proof of the main theorem (Theorem 6.1).

Synopsis. Following the introduction of monoidal, symmetric monoidal and cartesian presentations together with the related notions of PROs, PROPs and Lawvere categories in Section 2, we recall basic definitions and results on distributive laws of PROs and PROPs from [31] and [43] in Section 3. As mathematical objects, distributive laws are amenable to some operations illustrated in Section 4 - that are a tool-kit for proving our results: in Section 5. we show the distributive law of PROs that gives rise to the composite $\mathcal{T}_{\Sigma, E}$ and, in Section 6, the distributive law of PROPs that yields $\mathcal{L}_{\Sigma, E}$.

Prerequisites and notation. We assume familiarity with the basics of category theory (see e.g. [35, 15]), the definition of symmetric strict monoidal category [35, 39] (often abbreviated as SMC) and of bicategory [15, 4]. We write $f ; g: a \rightarrow c$ for composition of $f: a \rightarrow b$ and $g: b \rightarrow c$ in a category $\mathbb{C}$, and $\mathbb{C}[a, b]$ for the hom-set of arrows $a \rightarrow b$. It will be sometimes convenient to indicate an arrow $f: a \rightarrow b$ of $\mathbb{C}$ as $x \stackrel{f \in \mathbb{C}}{\longrightarrow} y$ or also $\stackrel{\in \mathbb{C}}{\longrightarrow}$, if names are immaterial. For $\mathbb{C}$ an $\mathrm{SMC}, \oplus$ is its monoidal product, with unit object $I$, and $\sigma_{a, b}: a \oplus b \rightarrow b \oplus a$ is the symmetry associated with $a, b \in \mathbb{C}$. Given $\mathcal{F}: \mathbb{C}_{1} \rightarrow \mathbb{C}_{2}$, we denote with $\mathcal{F}^{o p}: \mathbb{C}_{1}^{o p} \rightarrow \mathbb{C}_{2}^{o p}$ the induced functor on the opposite categories of $\mathbb{C}_{1}, \mathbb{C}_{2}$. 


$$
\begin{gathered}
\left(t_{1} ; t_{3}\right) \oplus\left(t_{2} ; t_{4}\right)=\left(t_{1} \oplus t_{2}\right) ;\left(t_{3} \oplus t_{4}\right) \\
\left(t_{1} ; t_{2}\right) ; t_{3}=t_{1} ;\left(t_{2} ; t_{3}\right) \quad i d_{n} ; c=c=c ; i d_{m} \\
\left(t_{1} \oplus t_{2}\right) \oplus t_{3}=t_{1} \oplus\left(t_{2} \oplus t_{3}\right) \quad i d_{0} \oplus t=t=t \oplus i d_{0}
\end{gathered}
$$

Figure 1: Axioms of strict monoidal categories for a PRO.

$$
\sigma_{1,1} ; \sigma_{1,1}=i d_{2} \quad\left(t \oplus i d_{z}\right) ; \sigma_{m, z}=\sigma_{n, z} ;\left(i d_{z} \oplus t\right)
$$

Figure 2: Extra axioms describing the symmetries in a PROP.

\section{Monoidal, Symmetric Monoidal and Cartesian Theories}

\subsection{Monoidal presentations and PROs}

Our exposition begins with monoidal presentations: specifications for algebraic structures borne by objects in a monoidal category. The associated categorical notion is called PRO (monoidal product category, after [34]).

Definition 2.1. A (one-sorted) monoidal presentation $(M P)$ is a pair $(\Sigma, E)$ consisting of a signature $\Sigma$ and a set of equations $E$. The signature $\Sigma$ is a set of generators $o: n \rightarrow m$, where $m$ and $n$ are natural numbers, $n$ is the arity, and $m$ the coarity. The set of monoidal $\Sigma$-terms is obtained by composing generators in $\Sigma$ and the unit $i d: 1 \rightarrow 1$ with; and $\oplus$. This is a purely formal process: given $\Sigma$-terms $t: k \rightarrow l, u: l \rightarrow m, v: m \rightarrow n$, one constructs new $\Sigma$-terms $t ; u: k \rightarrow m$ and $t \oplus v: k+n \rightarrow l+n$. The set $E$ of equations contains pairs $\left(t, t^{\prime}: n \rightarrow m\right)$ of monoidal $\Sigma$-terms with the same arity and coarity.

A monoidal presentation is said to be operadic when all the operations have coarity 1 and all equations are between $\Sigma$-terms of coarity 1.

A $\mathrm{PRO}$ is a strict monoidal category with objects the natural numbers, where $\oplus$ on objects is addition. Morphisms between PROs are strict identityon-objects monoidal functors: PROs and their morphisms form the category PRO. We call a sub-PRO a sub-category of a PRO which is also a PRO, with the inclusion functor a PRO morphism.

The PRO $\mathrm{S}_{(\Sigma, E)}$ freely generated by an $M P(\Sigma, E)$ has as its set of arrows $n \rightarrow m$ the set of monoidal $\Sigma$-terms $n \rightarrow m$ taken modulo the laws of strict monoidal categories - Fig. 1 — and the smallest congruence (with respect to ; and $\oplus$ ) containing the equations $t=t^{\prime}$ for any $\left(t, t^{\prime}\right) \in E$. When $E$ is empty, we usually write $\mathrm{S}_{\Sigma}$ for the freely generated PRO. 
There is a natural graphical representation for arrows of a PRO as string diagrams, which we now sketch, referring to [39] for the details. A monoidal $\Sigma$-term $n \rightarrow m$ is pictured as a box with $n$ ports on the left and $m$ ports on the right. Composition via ; and $\oplus$ are rendered graphically by horizontal and vertical juxtaposition of boxes, respectively.

$$
t ; s \text { is drawn } t=
$$

In any MP there are specific $\Sigma$-terms generating the underlying symmetric monoidal structure: these are $i d_{1}: 1 \rightarrow 1$, represented as $\square$, and the unit object for $\oplus$, that is, $i d_{0}: 0 \rightarrow 0$, whose representation is an empty diagram $\square$. Graphical representation for arbitrary identities $i d_{n}$ are generated according to the pasting rules in (1).

\section{Example 2.2.}

(a) We consider the monoidal presentation based on a the signature with a

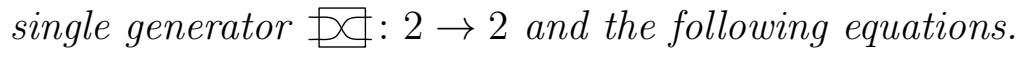
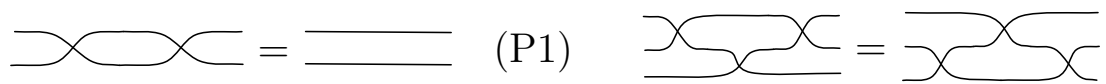

Write $\mathrm{P}$ for the corresponding freely generated PRO. There is a concrete description of the string diagrams of $\mathrm{P}$ as permutations: $\mathrm{P}$ is isomorphic to the PRO whose arrows $n \rightarrow m$ exist only when $n=m$, in which case they are the permutation on the $n$-elements set.

(b) The $\operatorname{MP}\left(\Sigma_{M}, E_{M}\right)$ of monoids is based on a signature $\Sigma_{M}$ with a multiplication $\mathrm{D}_{\mathrm{O}}: 2 \rightarrow 1$ and $a$ unit $0-0 \rightarrow 1$. The set of equations $E_{M}$ asserts associativity (A1) and unitality (A2)-A3 of the multiplication.
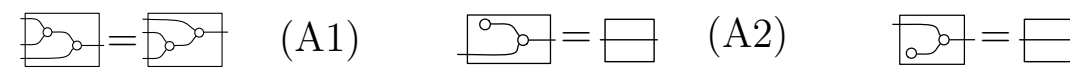

We write $\mathrm{Mn}$ for the corresponding freely generated PRO. Mn also has a concrete description, as the PRO D whose arrows $n \rightarrow m$ are order preserving functions between finite ordinals $\{0<\cdots<n-1\}$ and $\{0<\cdots<m-1\}$. We have $\mathrm{Mn} \cong \mathrm{D}$, see [31].

(c) We also introduce the $M P\left(\Sigma_{C}, E_{C}\right)$ of comonoids. The signature $\Sigma_{C}$ consists of a comultiplication $\_: 1 \rightarrow 2$ and a counit $\bullet: 1 \rightarrow 0$. The equations in $E_{C}$ are as follows. 

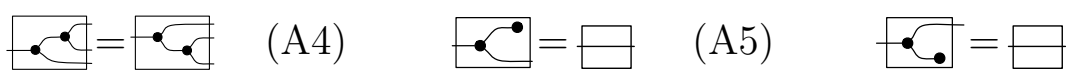

We name $\mathrm{Cm}$ the PRO freely generated by the presentation of comonoids. Modulo the white vs. black colouring, string diagrams of $\mathrm{Cm}$ can be seen as those of $\mathrm{Mn}$ "reflected about the y-axis". This observation yields $\mathrm{Cm}=\mathrm{Mn}^{o p}$.

Remark 2.3 (Models of a PRO). The assertion that $\left(\Sigma_{M}, E_{M}\right)$ is the MP of monoids - and similarly for other MPs appearing in our exposition-can be made precise using the notion of model (sometimes also called algebra) of a PRO. Given a strict monoidal category $\mathbb{C}$, a model of a PRO $\mathbf{S}$ in $\mathbb{C}$ is a strict monoidal functor $\mathcal{F}: S \rightarrow \mathbb{C}$. Then $\operatorname{Lin} \operatorname{Mod}(\mathrm{S}, \mathbb{C})$ is the category of models of $\mathbf{S}$ in $\mathbb{C}$ and natural transformations between them.

Turning to monoids, there is a category Monoid $(\mathbb{C})$ whose objects are the monoids in $\mathbb{C}$, i.e., objects $x \in \mathbb{C}$ equipped with arrows $x \oplus x \rightarrow x$ and $I \rightarrow x$, satisfying the usual equations. Given any model $\mathcal{F}: \mathrm{Mn} \rightarrow \mathbb{C}$, it follows that $\mathcal{F}(1)$ is a monoid in $\mathbb{C}$ : this yields a functor $\operatorname{LinMod}(\mathrm{Mn}, \mathbb{C}) \rightarrow \operatorname{Monoid}(\mathbb{C})$. Saying that $\left(\Sigma_{M}, E_{M}\right)$ is the MP of monoids means that this functor is an equivalence natural in $\mathbb{C}$.

Similar considerations hold for symmetric and cartesian presentations, introduced below, for which one takes as models symmetric monoidal and cartesian categories respectively. We refer the reader to [24, 31] for more information.

\subsection{Symmetric Monoidal presentations and PROPs}

We now introduce symmetric monoidal presentations, describing algebraic structures borne by objects in a symmetric monoidal category. Differently from monoidal presentations, they come with a built-in, natural symmetry $\mathcal{C}$, that allows one to express the "swapping" of variables and thus commutativity of operations. The corresponding categorical notion is the one of PROP (monoidal product and permutation category [34]): a PROP is just a $\mathrm{PRO}$ which, as a strict monoidal category, is equipped with a symmetric structure.

Definition 2.4. A (one-sorted) symmetric monoidal presentation (SMP) is a pair $(\Sigma, E)$ consisting of a signature $\Sigma$ and a set of equations $E$. The difference from a monoidal presentation is that in $E$ symmetric monoidal 
$\Sigma$-terms appear: these are obtained by composing generators in $\Sigma$, the unit

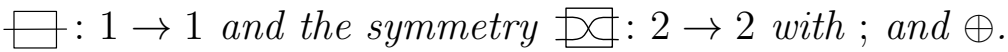

As for MPs, an SMP is said to be operadic when all the operations have coarity 1 and all the equations only involve terms of coarity 1.

A PROP is a symmetric strict monoidal category with objects the natural numbers, where $\oplus$ on objects is addition. Morphisms between PROPs are strict symmetric identity-on-objects monoidal functors: PROPs and their morphisms form the category PROP. We call a sub-PROP a sub-category of a PROP that is a PROP with the inclusion functor being a PROP morphism.

The PROP $\mathcal{T}_{(\Sigma, E)}$ freely generated by an $\operatorname{SMP}(\Sigma, E)$ has as its set of arrows $n \rightarrow m$ symmetric monoidal $\Sigma$-terms $n \rightarrow m$ modulo the laws of symmetric strict monoidal categories - Fig. 1 and 2 - and the smallest congruence (w.r.t. ; and $\oplus$ ) containing equations $t=t^{\prime}$ for any $\left(t, t^{\prime}\right) \in E$.

\section{Example 2.5.}

(a) The PRO P of permutations (Ex. 2.2 (a)) is also a PROP: the unique generator $\mathrm{D}$ in the associated monoidal presentation defines the symmetry of type $1+1 \rightarrow 1+1$ and can be used to define arbitrary symmetries $n+m \rightarrow m+n$. Equations (P1)-( definition satisfies the laws of symmetric monoidal categories (Fig. 2).

Observe that, as a PROP, $\mathrm{P}$ is freely generated by the empty symmetric monoidal presentation $(\emptyset, \emptyset)$. $\mathrm{P}$ is the initial object in $\mathbf{P R O P}$.

(b) The SMP of commutative monoids is defined by the same signature $\Sigma_{M}$ as the MP of monoids (Ex. 2.2.(b)). The set of equations $E_{C M}$ is given by those $E_{M}$ of monoids with the addition of the following commutativity equation:

$$
D_{0}=\mathrm{pO}^{\circ} \text {. }
$$

Note the use of the symmetry in the right-hand side of (A7). We name CMn the PROP freely generated by the SMP of commutative monoids. It is isomorphic to the PROP F whose arrows $n \rightarrow m$ are functions from $\bar{n}$ to $\bar{m}$, where $\bar{z}:=\{0,1, \ldots, z-1\}$.

(c) Dually, one can freely generate the PROP CCm of commutative comonoids from the signature $\Sigma_{C}$ of comonoids (Ex. 2.2.(c)) and a set of equations 
$E_{C C}$ given by the equations $E_{C}$ of comonoids plus the following:

$$
\sigma=\varnothing
$$

As in the monoidal case, we have $\mathrm{CCm} \cong \mathrm{CMn}^{\text {op }}$ and also $\mathrm{CCm} \cong \mathrm{F}^{o p}$.

(d) The PROP B of (commutative/cocommutative) bialgebras is generated by the SMP based on signature $\Sigma_{M} \uplus \Sigma_{C}$ and equations $E_{C M} \uplus E_{C C} \uplus B$, where $B$ is as follows:

$$
\begin{aligned}
& \bar{D} \bullet= \\
& \stackrel{a c}{a}=\begin{array}{l}
0 \\
0
\end{array}
\end{aligned}
$$

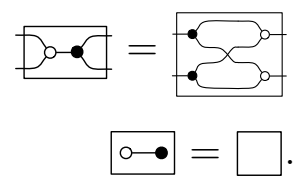

Remark 2.6. Notice that one can always mimic an $S M P(\Sigma, E)$ with an appropriate $M P\left(\Sigma^{\prime}, E^{\prime}\right)$, such that the free $P R O P \mathcal{T}_{(\Sigma, E)}$ is isomorphic to the free PRO $\mathrm{S}_{\left(\Sigma^{\prime}, E^{\prime}\right)}$. Suppose for instance to aim at the monoidal presentation of commutative monoids. Differently from the SMP, now the symmetry $\Phi$ must be explicitly added to the signature, which becomes $\left\{D_{0}, \square, \infty\right\}$. Moreover, one needs to add to (A1), (A2) and (A7) equations that guarantee naturality of the symmetric structure: these will be (P1), (Iי) and

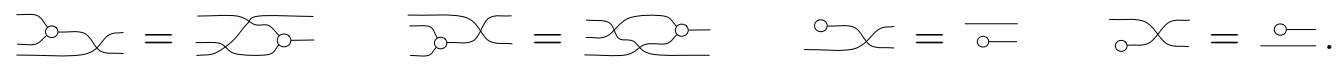

One can verify that the PRO freely generated by this $M P$ is the same as the PROP CMn of Example 2. D(b). This observation suggests a generic construction of PROPs in terms of PROs "interacting with a symmetry": we shall make this precise in Section 5, using the concept of distributive law of PROs (Section 3).

\subsection{Cartesian presentations and Lawvere Categories}

An additional layer of structure is introduced when passing from symmetric monoidal to cartesian presentations, adapted to describe algebraic structures borne by objects in a cartesian category. The associated categorical concept is the one of Lawvere category [33, 29].

Definition 2.7. A (one-sorted) cartesian presentation $(\Sigma, E)$ consists of a signature $\Sigma=\left\{o_{1}: n_{1} \rightarrow 1, \ldots, o_{k}: n_{k} \rightarrow 1\right\}$ and a set $E$ of equations between cartesian $\Sigma$-terms, which are defined as follows: 
- for each $i \in \mathbb{N}$, the variable $x_{i}$ is a cartesian term;

- suppose $o: n \rightarrow 1$ is a generator in $\Sigma$ and $t_{1}, \ldots, t_{n}$ are cartesian terms. Then $o\left(t_{1}, \ldots, t_{n}\right)$ is a cartesian term.

A Lawvere category is a cartesian category (i.e., with finite products) whose objects are the natural numbers. A cartesian presentation $(\Sigma, E)$ freely generates a Lawvere category $\mathcal{L}_{(\Sigma, E)}$ as follows. Arrows $n \rightarrow m$ are lists $\left\langle t_{1}, \ldots, t_{m}\right\rangle$ of cartesian $\Sigma$-terms quotiented by $E$, such that, for each $t_{i}$, only variables among $x_{1}, \ldots, x_{n}$ appear in $t_{i}$. Composition is by substitution:

$$
\left(n \stackrel{\left\langle t_{1}, \ldots, t_{m}\right\rangle}{\longrightarrow} m\right) ;\left(m \stackrel{\left\langle s_{1}, \ldots, s_{z}\right\rangle}{\longrightarrow} z\right)=n \stackrel{\left\langle s_{1}\left[t_{i} / x_{i} \mid 1 \leq i \leq m\right], \ldots, s_{z}\left[t_{i} / x_{i} \mid 1 \leq i \leq m\right]\right\rangle}{\longrightarrow} z
$$

where $t\left[t^{\prime} / x\right]$ denotes the cartesian term $t$ in which all occurrences of the variable $x$ have been replaced with $t^{\prime}$.

The product $\times$ in $\mathcal{L}_{(\Sigma, E)}$ is defined on objects by addition and on arrows by list concatenation and suitable renaming of variables:

$$
\begin{aligned}
\left(n \stackrel{\left\langle t_{1}, \ldots, t_{m}\right\rangle}{\longrightarrow} m\right) & \times\left(z \stackrel{\left\langle s_{1}, \ldots, s_{l}\right\rangle}{\longrightarrow} l\right) \\
& =n+z \stackrel{\left\langle t_{1}, \ldots, t_{m}, s_{1}\left[x_{i+m} / x_{i} \mid 1 \leq i \leq z\right], \ldots, s_{l}\left[x_{i+m} / x_{i} \mid 1 \leq i \leq z\right]\right\rangle}{\longrightarrow} m+l .
\end{aligned}
$$

We use notation ovar $(t)$ for the list of occurrences of variables appearing (from left to right) in $t$ and, more generally, $\operatorname{ovar}\left(t_{1}, \ldots, t_{m}\right)$ for the list $\operatorname{ovar}\left(t_{1}\right):: \cdots:: \operatorname{ovar}\left(t_{m}\right)$. Also, $|l| \in \mathbb{N}$ denotes the length of a list $l$. We say that a list $\left\langle t_{1}, \ldots, t_{m}\right\rangle: n \rightarrow m$ is linear if each variable among $x_{1}, \ldots, x_{n}$ appears exactly once in $\operatorname{ovar}\left(t_{1}, \ldots, t_{m}\right)$.

Any Lawvere category is also a PROP, with the product inducing a symmetric monoidal structure. For a freely generated Lawvere category $\mathcal{L}_{(\Sigma, E)}$, the symmetry $n+m \rightarrow m+n$ is defined as the list $\left\langle x_{n+1}, \ldots, x_{n+m}, x_{1}, \ldots, x_{n}\right\rangle$. Moreover, note that the unique PROP morphism $\mathrm{P} \rightarrow \mathcal{L}_{(\Sigma, E)}$ given by initiality of $\mathrm{P}$ in $\mathbf{P R O P}$ sends a permutation $p: n \rightarrow n$ to $\left\langle x_{p^{-1}(1)}, \ldots, x_{p^{-1}(n)}\right\rangle$.

Example 2.8. The $\operatorname{SMP}\left(\Sigma_{M}, E_{C M}\right)$ of commutative monoids can be also thought of as a cartesian presentation. Indeed all the operations in $\Sigma_{M}$ have coarity 1 and $E_{C M}$ only involves cartesian $\Sigma$-terms built from them, e.g. (A2) becomes $\mathrm{Do}\left(\mathrm{o}, x_{1}\right)=x_{1}$ and (A7) becomes $\mathrm{Do}\left(x_{2}, x_{1}\right)=\mathrm{Do}\left(x_{1}, x_{2}\right)$. The presentation $\left(\Sigma_{M}, E_{C M}\right)$ generates the Lawvere category $\mathcal{L}_{\Sigma_{M}, E_{M}}$ whose arrows $n \rightarrow m$ are lists $\left\langle t_{1}, \ldots, t_{m}\right\rangle$ of elements of the free commutative monoid on $\left\{x_{1}, \ldots, x_{n}\right\}$. 
Remark 2.9. Contrarily to what Example 2.8 may suggest, cartesian presentations are not a subclass of symmetric monoidal presentations: in fact, the two are incomparable. On the one hand, a symmetric monoidal presentation $(\Sigma, E)$ is cartesian if and only if all generators in $\Sigma$ have coarity 1 and, for all equations $t=s$ in $E, t$ and $s$ are $\Sigma$-terms with coarity 1 . Under these conditions, there is a canonical way to interpret any $\Sigma$-term $n \rightarrow m$ as a list of $m$ cartesian $\Sigma$-terms on variables $x_{1}, \ldots, x_{n}$. Below, $=-$ ranges over $\Sigma$ :

$$
\begin{aligned}
& \square: 1 \rightarrow 1 \mapsto\left\langle x_{1}\right\rangle \quad \quad \bar{\varnothing}: 2 \rightarrow 2 \mapsto\left\langle x_{2}, x_{1}\right\rangle \\
& \overline{\overline{\mathrm{i}}} \boldsymbol{O}-\mathrm{n} \rightarrow 1 \mapsto\left\langle\overline{\mathrm{i}} \boldsymbol{O}-\left(x_{1}, \ldots, x_{n}\right)\right\rangle .
\end{aligned}
$$

The inductive cases are defined using the operations ; and $\oplus$ on lists given in Definition 2.7. Note that $\Sigma$-terms always denote (lists of) linear cartesian terms. This explains why, conversely, not all the cartesian presentations are symmetric monoidal: their equations possibly involve non-linear $\Sigma$-terms, which are not expressible with (symmetric monoidal) $\Sigma$-terms. The subtlety here is that, in a sense, we can still simulate a cartesian presentation on signature $\Sigma$ with a symmetric monoidal presentation, which however will be based on a larger signature $\Sigma^{\prime}$, recovering the possibility of copying and discarding variables by the use of additional generators. This is analogous with the "encoding" of symmetric monoidal presentations in monoidal presentations, described in Remark 2.6. It will be made precise in Section 6, using distributive laws of PROPs introduced in the next section.

\section{Distributive Laws}

A basic operation on monoidal presentations $(\Sigma, E)$ and $\left(\Sigma^{\prime}, E^{\prime}\right)$ is their sum $\left(\Sigma \uplus \Sigma^{\prime}, E \uplus E^{\prime}\right)$. In PRO, the PRO generated by $\left(\Sigma \uplus \Sigma^{\prime}, E \uplus E^{\prime}\right)$ is the coproduct $\mathcal{T}_{(\Sigma, E)}+\mathcal{T}_{\left(\Sigma^{\prime}, E^{\prime}\right)}$ of the two freely generated PROs.

The sum $\mathcal{T}_{(\Sigma, E)}+\mathcal{T}_{\left(\Sigma^{\prime}, E^{\prime}\right)}$ is the least interesting way of combining presentations, because there are no equations that express compatibility between the algebraic structures in $(\Sigma, E)$ and $\left(\Sigma^{\prime}, E^{\prime}\right)$. Interactions between operations is a standard pattern in algebra: e.g. a ring is given by a monoid and an abelian group, subject to equations that ensure that the former distributes over the latter. Similarly, the equations of bialgebras (Example 2.5 (d) describe the interplay of a commutative monoid and a cocommutative comonoid. Functions between finite sets, which can always be decomposed as a surjection followed by an injection, are another example. 
In [31] Lack shows how these phenomena can be uniformly described as a composition operation, definable both for PROs and PROPs 3 . The conceptual switch is to understand these categories as monads, and the composition operation as a distributive law. The monads are the objects of a certain bicategory [4], as in the classical work of Street [42]

Definition 3.1. A monad on an object $x$ of a bicategory $\mathfrak{B}$ is a 1-cell $\mathcal{F}: x \rightarrow x$ with 2-cells $\eta^{\mathcal{F}}: i d_{x} \rightarrow \mathcal{F}$ and $\mu^{\mathcal{F}}: \mathcal{F} ; \mathcal{F} \rightarrow \mathcal{F}$ (called the unit and the multiplication respectively) making the following diagrams-in which we suppress the associativity isomorphisms-commute.
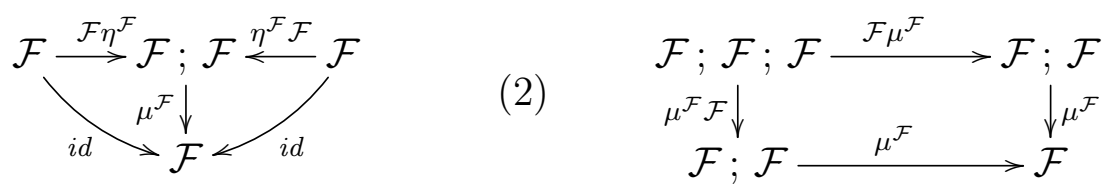

A morphism between monads $x \stackrel{\mathcal{F}}{\rightarrow} x$ and $x \stackrel{\mathcal{G}}{\rightarrow} x$ is a 2 -cell $\theta: \mathcal{F} \rightarrow \mathcal{G}$ making the following diagrams commut $\S^{5}$.
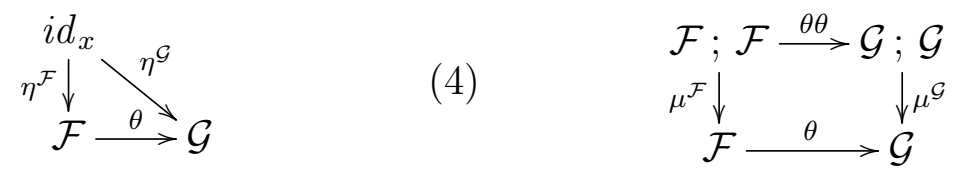

An epimorphic monad morphism is called a monad quotient.

For $\mathfrak{B}=$ Cat, the above definition yields the standard notion of monad as an endofunctor with a pair of natural transformations.

Something interesting happens for the case of the bicategory $\mathfrak{B}=\operatorname{Span}($ Set) whose objects are sets, 1 -cells are spans $A \stackrel{f}{\leftarrow} B \stackrel{g}{\rightarrow} C$ of functions with composition defined by pullback

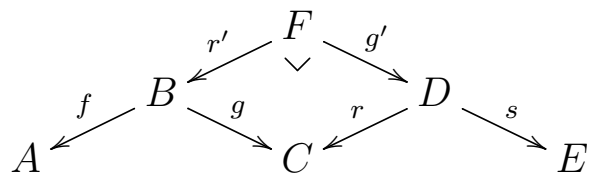

\footnotetext{
${ }^{3}$ One can use the same techniques to define composition of Lawvere categories, see [19]. We will not cover this case as it is not needed for our developments.

${ }^{4}$ Actually, Street worked in a 2-category, but the same presentation can be developed in any bicategory with the obvious, minor modifications [31, §3.1].

${ }^{5} \mathrm{~A}$ notion of morphism can be defined also between monads on different objects, like in [42. We will not need that level of generality here.
} 
and 2-cells are span morphisms, i.e. functions $\alpha: B \rightarrow B^{\prime}$ making the two triangles commute:

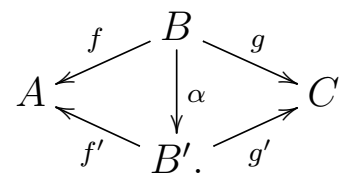

Monads in Span(Set) are precisely the small categories. Indeed, a monad $(\mathcal{F}, \eta, \mu)$ in $\operatorname{Span}($ Set $)$ consists of an endospan $O b \stackrel{\text { dom }}{\longleftarrow} A r \stackrel{\text { cod }}{\longleftrightarrow} O b$, where $O b$ serves as the set of objects, and $A r$ as the set of arrows. The remaining data in the span is the domain/codomain maps $A r \rightrightarrows O b$. The unit $\eta: i d \rightarrow \mathcal{F}$ is a span morphism associating an identity arrow to each object (below left). The multiplication $\mu: \mathcal{F} ; \mathcal{F} \rightarrow \mathcal{F}$ is a span morphism defining composition for any two arrows $a \stackrel{f}{\rightarrow} b \stackrel{g}{\rightarrow} c$ in $A r$ (below right).
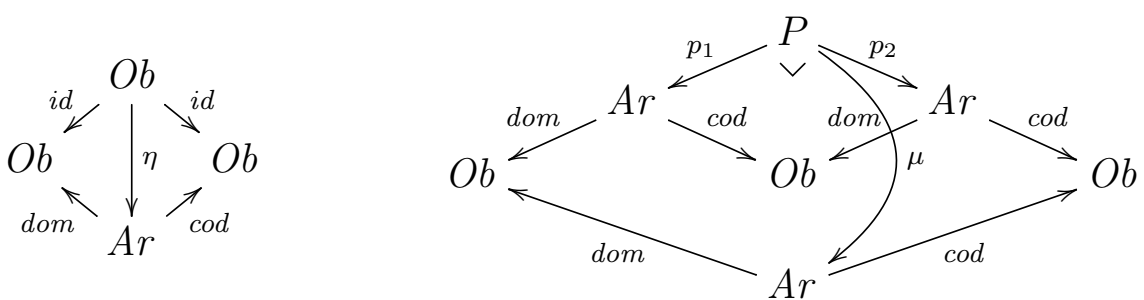

By thinking of categories as monads, one can define the composition of categories with the same set of objects as monad composition by a distributive law in Span(Set). This phenomenon is studied in [37].

Definition 3.2. Let $\left(\mathcal{F}, \eta^{\mathcal{F}}, \mu^{\mathcal{F}}\right),\left(\mathcal{G}, \eta^{\mathcal{G}}, \mu^{\mathcal{G}}\right)$ be monads in a bicategory $\mathfrak{B}$ on the same object. A distributive law of $\mathcal{F}$ over $\mathcal{G}$ is a 2 -cell $\lambda: \mathcal{F} ; \mathcal{G} \rightarrow \mathcal{G} ; \mathcal{F}$ in $\mathfrak{B}$ making the following - in which we again omit associativity-commute.
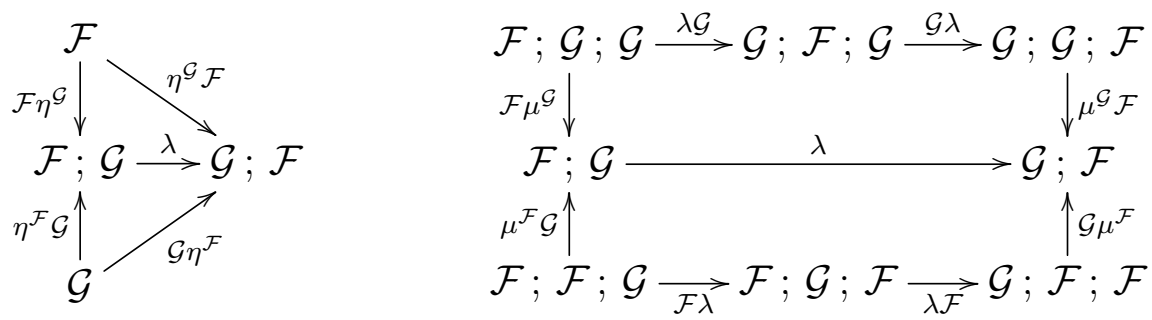

$A$ distributive law $\lambda: \mathcal{F} ; \mathcal{G} \rightarrow \mathcal{G} ; \mathcal{F}$ yields a monad $\mathcal{G} ; \mathcal{F}$ with the following 
unit and multiplication:

$$
\begin{aligned}
& \eta^{\mathcal{G} ; \mathcal{F}}: \quad i d \stackrel{\eta^{\mathcal{F}}}{\longrightarrow} \mathcal{F} \stackrel{\eta^{\mathcal{G}} \mathcal{F}}{\longrightarrow} \mathcal{G} ; \mathcal{F} \\
& \mu^{\mathcal{G} ; \mathcal{F}}: \quad \mathcal{G} ; \mathcal{F} ; \mathcal{G} ; \mathcal{F} \stackrel{\mathcal{G} \lambda \mathcal{F}}{\longrightarrow} \mathcal{G} ; \mathcal{G} ; \mathcal{F} ; \mathcal{F} \stackrel{\mu^{\mathcal{G}} \mathcal{F} \mathcal{F}}{\longrightarrow} \mathcal{G} ; \mathcal{F} ; \mathcal{F} \stackrel{\mathcal{G} \mu^{\mathcal{F}}}{\longrightarrow} \mathcal{G} ; \mathcal{F}
\end{aligned}
$$

Let us verify how the abstract definition works for the case of categories. Pick categories $\mathbb{C}$ and $\mathbb{D}$ with the same set $O b$ of objects, seen as monads $O b \stackrel{\operatorname{dom}_{\mathbb{C}}}{\longleftarrow} A r_{\mathbb{C}} \stackrel{\operatorname{cod}_{\mathbb{C}}}{\longleftrightarrow} O b$ and $O b \stackrel{\operatorname{dom}_{\mathbb{D}}}{\longleftarrow} A r_{\mathbb{D}} \stackrel{\operatorname{cod}_{\mathbb{D}}}{\longrightarrow} O b$ in Span(Set). A distributive law $\lambda: \mathbb{C} ; \mathbb{D} \rightarrow \mathbb{D} ; \mathbb{C}$ is a span morphism

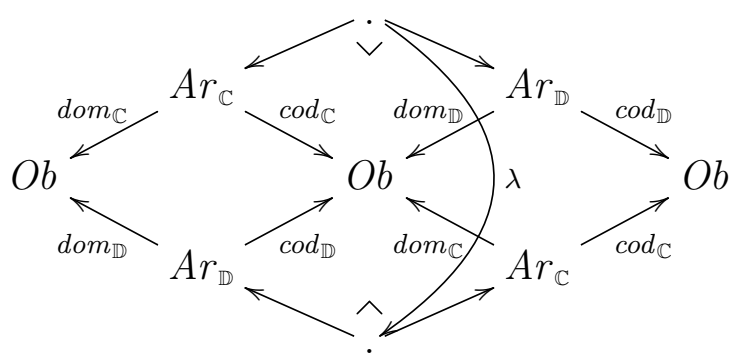

mapping composable pairs $a \stackrel{\in \mathbb{C}}{\longrightarrow} \stackrel{\in \mathbb{D}}{\longrightarrow} b$ to composable pairs $a \stackrel{\in \mathbb{D}}{\longrightarrow} \stackrel{\in \mathbb{C}}{\longrightarrow} b$. As described in (7), $\lambda$ allows to define a monad structure on $\mathbb{D} ; \mathbb{C}$. That means, $\lambda$ yields a category $\mathbb{D} ; \mathbb{C}$ whose arrows $a \rightarrow b$ are composable pairs $a \stackrel{\in \mathbb{D}}{\longrightarrow} \stackrel{\in C}{\longrightarrow} b$ of arrows of $\mathbb{D}, \mathbb{C}$ and composition is defined as

$$
(a \stackrel{f \in \mathbb{D}}{\longrightarrow} \stackrel{g \in \mathbb{C}}{\longrightarrow} b) ;\left(b \stackrel{f^{\prime} \in \mathbb{D}}{\longrightarrow} \stackrel{g^{\prime} \in \mathbb{C}}{\longrightarrow} c\right) \quad:=\quad\left(a \stackrel{f \in \mathbb{D}}{\longrightarrow} \lambda\left(\stackrel{g \in \mathbb{C}}{\longrightarrow} \stackrel{f^{\prime} \in \mathbb{D}}{\longrightarrow}\right) \stackrel{g^{\prime} \in \mathbb{C}}{\longrightarrow} c\right) .
$$

Composition of PROs works analogously to composition of categories. In order to take into account the monoidal structure, one need to pass from Span(Set) to Span(Mon), whose objects are monoids and 1-cells are spans in the category Mon of monoids. Monads in Span(Mon) are small strict monoidal categories and thus PROs are monads on the monoid $\mathbb{N}$ of natural numbers. Composing two PROs via a distributive law again yields a PRO.

The case of PROPs requires yet another refinement, in order to take into account the symmetries. An appropriate setting that achieves this is the bicategory Bimod(Span(Mon)) whose objects are small strict monoidal categories and 1-cells are the bimodules in Span(Mon). PROPs are monads on the object $\mathrm{P}$ of $\operatorname{Bimod}(\operatorname{Span}($ Mon $))$. 
We shall gloss over further details, as they are out of the scope of this paper - we refer to [31] and [43, § 2.4] for an extensive treatment. The simpler setting of composition of mere categories should provide enough guidance to follow the rest of our exposition.

It is important for our purposes to remark how composition works for PROs $S_{\Sigma, E}, S_{\Sigma^{\prime}, E^{\prime}}$ generated by MPs, say $(\Sigma, E)$ and $\left(\Sigma^{\prime}, E^{\prime}\right)$. The PRO $\mathrm{S}_{\Sigma, E} ; \mathrm{S}_{\Sigma^{\prime}, E^{\prime}}$ induced by a distributive law $\lambda: \mathrm{S}_{\Sigma^{\prime}, E^{\prime}} ; \mathrm{S}_{\Sigma, E} \rightarrow \mathrm{S}_{\Sigma, E} ; \mathrm{S}_{\Sigma^{\prime}, E^{\prime}}$ will also enjoy a presentation by generators and equations, consisting of the sum $\left(\Sigma \uplus \Sigma^{\prime}, E \uplus E^{\prime}\right)$ plus the equations $E_{\lambda}$ arising from the the distributive law. The set $E_{\lambda}$ is simply the graph of $\lambda$, now seen as a set of directed equations $\left(\stackrel{\in \mathrm{S}_{\Sigma^{\prime}, E^{\prime}}}{\longrightarrow} \stackrel{\in \mathrm{S}_{\Sigma, E}}{\longrightarrow}\right) \approx\left(\stackrel{\in \mathrm{S}_{\Sigma, E}}{\longrightarrow} \stackrel{\in \mathrm{S}_{\Sigma^{\prime}, E^{\prime}}}{\longrightarrow}\right)$ telling how monoidal $\Sigma^{\prime}$-terms modulo $E^{\prime}$ distribute over monoidal $\Sigma$-terms modulo $E$. In fortunate cases, it is possible to present $E_{\lambda}$ by a simpler, or even finite, set of equations, thus giving a sensible axiomatisation of the compatibility conditions expressed by $\lambda$. This is the case for both examples considered below.

\section{Example 3.3.}

(a) The PRO of $\mathrm{Mn}$ of monoids, or equivalently the PRO of $\mathrm{D}$ of finite ordinals and monotonic functions (Example 2. की(b)), can be seen as the composite $\mathrm{SGr} ; \mathrm{S}_{\circ \text {. }}$. Here $\mathrm{SGr}$ is the PRO of semigroups, freely generated by $\left(\left\{\mathrm{D}_{0}\right\},\{(\mathrm{A} 1)\}\right)$, whereas $\mathrm{S}_{\circ}$ is the PRO freely generated by $(\{\circ\}, \varnothing)$. The witnessing distributive law $\lambda: \mathrm{S}_{\square} ; \mathrm{SGr} \rightarrow \mathrm{SGr} ; \mathrm{S}_{\square}$ is described in [31, Example 3.13]: its associated set $E_{\lambda}$ of equations is finitely presentable by (A2) and (A3). These additional equations say that $\circ$ acts as the unit for the semigroup, which is then a monoid. This explains why $\mathrm{SGr} ; \mathrm{S}_{\square} \cong \mathrm{Mn}$.

(b) The PROP of monoids is none other than $\mathrm{P}$; $\mathrm{Mn}$, where $\mathrm{P}$ is the PRO of permutations (Example 2. D(a)). The underlying distributive law of PROs $\mathrm{Mn} ; \mathrm{P} \rightarrow \mathrm{P} ; \mathrm{Mn}$ - see [31, Example 3.14] - is finitely presentable by the four equations displayed in Remark 2.6. This is a special case of a more general situation described in Theorem 5.1: operadic monoidal presentations can be considered as symmetric monoidal through composition with $\mathrm{P}$, via a distributive law. The PROP of commutative monoids $\mathrm{CMn}$ can then be obtained by quotienting $\mathrm{P} ; \mathrm{Mn}$ with equation (A7). 
Analogous considerations hold for distributive laws between PROPs freely generated by SMPs. We illustrate a few examples below.

\section{Example 3.4.}

(a) There is a PROP counterpart of Example 3. D( (a). The PROP F of functions can be described as the composite of PROPs for surjections and injections. Let In be the PROP whose arrows $n \rightarrow m$ are injective functions from $\bar{n}$ to $\bar{m}$. The PROP Su of surjective functions is defined analogously. There is a distributive law $\lambda: \operatorname{In} ; \mathrm{Su} \rightarrow \mathrm{Su} ;$ In defined by epi-mono factorisation: it maps a composable pair $\stackrel{\in \ln }{\longrightarrow} \stackrel{\in \mathrm{Su}}{\longrightarrow}$ to a composable pair $\stackrel{\in \mathrm{Su}}{\longrightarrow} \stackrel{\in \ln }{\longrightarrow}$ [31]. The resulting PROP Su; In is isomorphic to $\mathrm{F}$ because any function in $\mathrm{F}$ can be uniquely factorised (up-to permutation) as a surjection followed by an injection. In more syntactic terms, using the isomorphism $\mathrm{F} \cong \mathrm{CMn}$, this result says that $\mathrm{CMn}$ is the composite $\mathrm{Mu} ; \mathrm{Un}$, where $\mathrm{Mu} \cong \mathrm{Su}$ is the PROP freely generated by the $\operatorname{SMP}(\{\mathrm{D}-\},\{(\mathrm{A} 1), \mathrm{A} 7 \mathrm{D}\})$ and $\mathrm{Un} \cong \mathrm{In}$ by the $\operatorname{SMP}(\{\mathrm{\circ}\}, \emptyset)$. The distributive law $\lambda: \ln ; \mathrm{Su} \rightarrow \mathrm{Su}$; In is then presented by the remaining equation (A2) of CMn, which indeed describes how the generator of of Un can be moved past the one $\mathrm{D}$ - of $\mathrm{Mu}$.

(b) The composition of $\mathrm{CCm}$ and $\mathrm{CMn}$ yields the PROP B of commutative bialgebras. First, because $\mathrm{CMn} \cong \mathrm{F}$ and $\mathrm{CCm} \cong \mathrm{F}^{o p}$, we can express a distributive law $\lambda: \mathrm{CMn} ; \mathrm{CCm} \rightarrow \mathrm{CCm} ; \mathrm{CMn}$ as having the type $\mathrm{F} ; \mathrm{F}^{o p} \rightarrow \mathrm{F}^{o p} ; \mathrm{F}$. This amounts to saying that $\lambda$ maps cospans $n \stackrel{f \in \mathrm{F}}{\longrightarrow} \stackrel{g \in \mathrm{F}}{\longleftarrow} m$ to spans $n \stackrel{p \in \mathrm{F}}{\longleftarrow} \stackrel{q \in \mathrm{F}}{\longrightarrow} m$. Defining this mapping via (chosen) pullback in $\mathrm{F}$ satisfies the conditions of distributive laws [31]. One can now read the equations arising by the distributive law from pullback squares in F. For instance, consider the pullback square in $\mathrm{F}$ below left. The second diagram is obtained from the pullback by applying the isomorphisms $\mathrm{F} \cong \mathrm{CMn}$ and $\mathrm{F}^{o p} \cong \mathrm{CCm}$, and illustrates an equation induced by the distributive law.

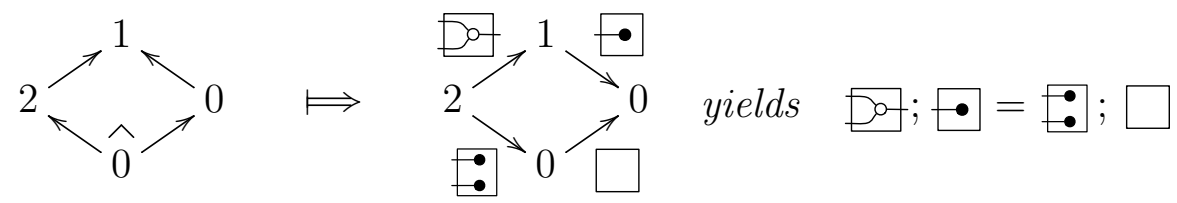

In fact, Lack [31] shows that the equations presenting CCm; CMn arise from (those of $\mathrm{CCm}+\mathrm{CMn}$ and) just four pullback squares, yielding 
equations $\mathrm{A} 9 \mathrm{~A}$ - $\mathrm{A} 12$. Therefore, $\mathrm{CCm}$; $\mathrm{CMn}$ is isomorphic to the PROP B of bialgebras encountered in Example 2.5 (d). Furthermore, these PROPs have a "concrete" descriptions as $\mathrm{F}^{o p} ; \mathrm{F}$. In the terminology of [4], one can see $\mathrm{F}^{o p}$; $\mathrm{F}$ as the classifying category of the bicategory Span $(\mathrm{F})$, obtained by identifying the isomorphic 1-cells and forgetting the 2-cells.

A convenient way of defining a distributive law is through a factorisation system. There is a tight relationship between the two notions: distributive laws of small categories are in 1-1 correspondence with so-called strict factorisation systems [37], in which factorisations must be specified uniquely on the nose, rather than merely up-to isomorphism. The same correspondence holds for distributive laws of PROs. Distributive laws of PROPs correspond instead to a more liberal kind of factorisation system, for which decompositions are up-to permutation. As this perspective will be useful later, we recall the following results from Lack [31].

Proposition 3.5 ([31], Theorem 3.8). Let $\mathrm{S}$ be a PRO and $\mathrm{S}_{1}, \mathrm{~S}_{2}$ be subPROs of S. Suppose that each arrow $n \stackrel{f \in \mathrm{S}}{\longrightarrow} m$ can be uniquely factorised as $n \stackrel{g_{1} \in \mathrm{S}_{1}}{\longrightarrow} \stackrel{g_{2} \in \mathrm{S}_{2}}{\longrightarrow} m$. Then there exists a distributive law of PROs $\lambda: \mathrm{S}_{2} ; \mathrm{S}_{1} \rightarrow$ $\mathrm{S}_{1} ; \mathrm{S}_{2}$, defined by associating to a composable pair $\stackrel{f \in \mathrm{S}_{2}}{\longrightarrow} \stackrel{g \in \mathrm{S}_{1}}{\longrightarrow}$ the factorisation of $f ; g$, yielding $\mathrm{S} \cong \mathrm{S}_{1} ; \mathrm{S}_{2}$.

Proposition 3.6 ([31], Theorem 4.6). Let $\mathcal{T}$ be a PROP and $\mathcal{T}_{1}, \mathcal{T}_{2}$ be sub-PROPs of $\mathcal{T}$. Suppose that each arrow $n \stackrel{f \in \mathcal{T}}{\longrightarrow} m$ can be factorised as $n \stackrel{g_{1} \in \mathcal{T}_{1}}{\longrightarrow} \stackrel{g_{2} \in \mathcal{T}_{2}}{\longrightarrow} m$ uniquely up-to permutation, that is, for any other decomposition $n \stackrel{h_{1} \in \mathcal{T}_{1}}{\longrightarrow} \stackrel{h_{2} \in \mathcal{T}_{2}}{\longrightarrow} m$ of $f$, there exists permutation $\stackrel{p \in \mathrm{P}}{\longrightarrow}$ such that the following diagram commutes.

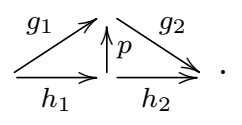

Then there exists a distributive law of PROPs $\lambda: \mathcal{T}_{2} ; \mathcal{T}_{1} \rightarrow \mathcal{T}_{1} ; \mathcal{T}_{2}$, defined by associating to a composable pair $\stackrel{f \in \mathcal{T}_{2}}{\longrightarrow} \stackrel{g \in \mathcal{T}_{1}}{\longrightarrow}$ the factorisation of $f ; g$, yielding $\mathcal{T} \cong \mathcal{T}_{1} ; \mathcal{T}_{2}$

\section{Operations on Distributive Laws}

This section illustrates various ways of manipulating distributive laws that will be useful in the sequel. 


\subsection{Quotient of a Distributive Law}

Definition 3.1 introduced the notion of quotient $\theta: \mathcal{F} \rightarrow \mathcal{G}$ of a monad $\mathcal{F}$ : an example of a monad quotient is the situation when the monad $\mathcal{G}$ is obtained by imposing additional equations on the presentation of $\mathcal{F}$. As one may expect, distributive laws are compatible with monad quotients, provided that the law respects the additional equations. This folklore result appears in various forms in the literature: [14] gives it for distributive laws of endofunctors over monads and [6, 13] for distributive laws of monads. All these references concern distributive laws in Cat. For our purposes, it is useful to state the result for arbitrary bicategories.

Proposition 4.1. Suppose that $\lambda: \mathcal{F} ; \mathcal{H} \rightarrow \mathcal{H} ; \mathcal{F}$ is a distributive law in a bicategory $\mathfrak{B}$.

(i) If $\theta: \mathcal{F} \rightarrow \mathcal{G}$ is a monad quotient and $\gamma: \mathcal{G} ; \mathcal{H} \rightarrow \mathcal{H} ; \mathcal{G}$ a 2-cell of $\mathfrak{B}$ making the following diagram commute

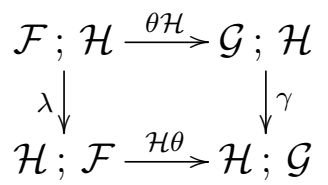

then $\gamma$ is a distributive law of monads.

(ii) If $\theta: \mathcal{H} \rightarrow \mathcal{M}$ is a monad quotient and $\delta: \mathcal{F} ; \mathcal{M} \rightarrow \mathcal{M} ; \mathcal{F}$ a 2-cell of $\mathfrak{B}$ making the following diagram commute

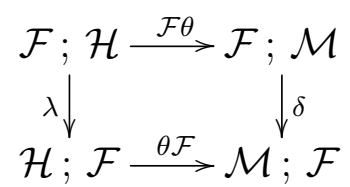

then $\delta$ is a distributive law of monads.

Proof. For statement (i), the diagrams for compatibility of $\gamma$ with unit and multiplication of $\mathcal{G}$ commute because $\theta$ is a monad morphism and (8) commutes. For compatibility of $\gamma$ with unit and multiplication of $\mathcal{H}$, one needs to use commutativity of (8) and the fact that $\theta$ is epi. The proof of statement (ii) is analogous. 
It is now useful to instantiate the result to the case of distributive laws of PROs and of PROPs. It turns out that it suffices to check compatibility of a distributive law with the equations determining the monad quotient.

Proposition 4.2. Let $\mathcal{T}, \mathcal{T}^{\prime}$ be PROs freely generated by $(\Sigma, E)$ and $\left(\Sigma, E^{\prime}\right)$ respectively, with $E \subseteq E^{\prime}$.

(i) Let $\lambda: \mathcal{T} ; \mathrm{S} \rightarrow \mathrm{S} ; \mathcal{T}$ be a distributive law such that, if $l=r$ is an equation of $E^{\prime}$, then $\lambda\left(\stackrel{i d_{n} \oplus l \oplus i d_{m} \in \mathcal{T}}{\longrightarrow} \stackrel{d \in \mathrm{S}}{\longrightarrow}\right)=\lambda\left(\stackrel{i d_{n} \oplus r \oplus i d_{m} \in \mathcal{T}}{\longrightarrow} \stackrel{d \in \mathrm{S}}{\longrightarrow}\right)$ for all $d, n$ and $m$. Then there exists a distributive law $\gamma: \mathcal{T}^{\prime} ; \mathbf{S} \rightarrow \mathbf{S} ; \mathcal{T}^{\prime}$ presented by the same equations as $\lambda$, i.e., $E_{\gamma}=E_{\lambda}$.

(ii) Let $\chi: S ; \mathcal{T} \rightarrow \mathcal{T}$; S be a distributive law such that, if $l=r$ is an equation of $E^{\prime}$, then $\chi\left(\stackrel{d \in \mathrm{S}}{\longrightarrow} \stackrel{i d_{n} \oplus l \oplus i d_{m} \in \mathcal{T}}{\longrightarrow}\right)=\chi\left(\stackrel{d \in \mathrm{S}}{\longrightarrow} \stackrel{i d_{n} \oplus r \oplus i d_{m} \in \mathcal{T}}{\longrightarrow}\right)$ for all $d, n$ and $m$. Then there exists a distributive law $\delta: \mathrm{S} ; \mathcal{T}^{\prime} \rightarrow \mathcal{T}^{\prime} ; \mathrm{S}$ presented by the same equations as $\chi$, i.e., $E_{\delta}=E_{\chi}$.

Both statements hold also starting from the PROPs freely generated by $(\Sigma, E)$, $\left(\Sigma, E^{\prime}\right)$ and distributive laws of PROPs.

Proof. We first focus on statement (i), splitting the proof in two parts. First, we show that, given our assumption about compatibility of $\lambda$ with $E^{\prime}$, the following, stronger statement follows:

if $c_{1}=c_{2}$ follows from the equations of $E^{\prime}$,

$$
\text { then } \lambda\left(\stackrel{c_{1} \in \mathcal{T}}{\longrightarrow} \stackrel{d \in \mathrm{S}}{\longrightarrow}\right)=\lambda\left(\stackrel{c_{2} \in \mathcal{T}}{\longrightarrow} \stackrel{d \in \mathrm{S}}{\longrightarrow}\right) \text { for all } d \text {. }
$$

In order to show (o), without loss of generality we can assume that $c_{1}=c_{2}$ holds because of the application of a single equation $l=r$ of $E^{\prime}$, that means,

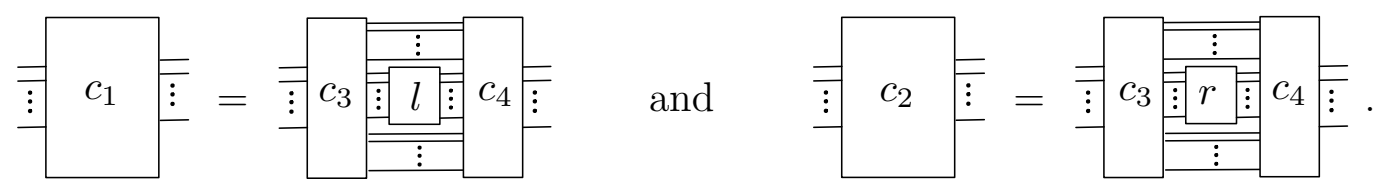

Because $\lambda$ is a distributive law of PROs, it preserves composition in $\mathcal{T}$ and S. That means, the computation of $\lambda\left(\stackrel{c_{1} \in \mathcal{T}}{\longrightarrow} \stackrel{d \in \mathrm{S}}{\longrightarrow}\right)$ goes componentwise: if

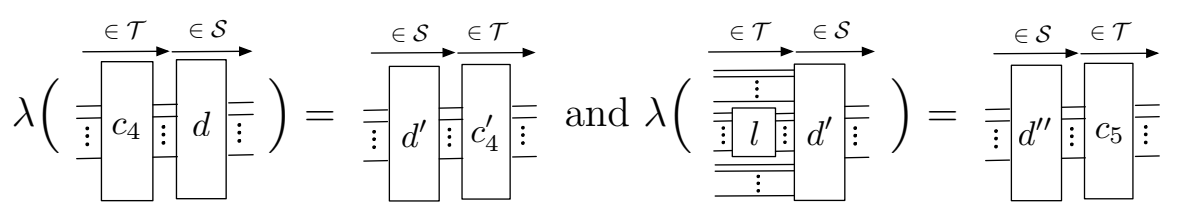


then

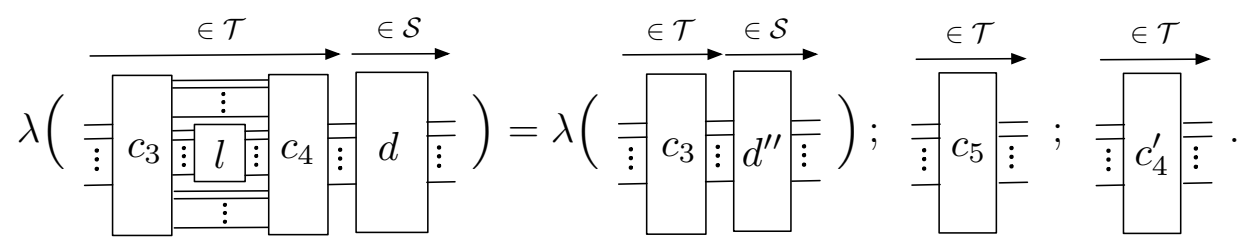

Because our assumption guarantees that $\lambda$ in 10 yields the same result with $r$ in place of $l$, we just proved (o).

We now exploit (o) to complete the proof (i). First, there is a PRO morphism $\theta: \mathcal{T} \rightarrow \mathcal{T}^{\prime}$ defined by quotienting string diagrams in $\mathcal{T}$ by $E^{\prime}$. This is a monad quotient in the bicategory Span(Mon) where PROs are monads. We now define another 2-cell $\gamma: \mathcal{T}^{\prime} ; \mathrm{S} \rightarrow \mathrm{S} ; \mathcal{T}^{\prime}$ as follows: given $\stackrel{e \in \mathcal{T}^{\prime}}{\longrightarrow} \stackrel{c \in \mathrm{S}}{\longrightarrow}$, pick any $\stackrel{d \in \mathcal{T}}{\longrightarrow}$ such that $\theta(d)=e$ and let $\stackrel{c^{\prime} \in \mathrm{S}}{\longrightarrow} \stackrel{d^{\prime} \in \mathcal{T}}{\longrightarrow}$ be $\lambda(\stackrel{d \in \mathcal{T}}{\longrightarrow} \stackrel{c \in \mathrm{S}}{\longrightarrow})$. Define $\gamma\left(\stackrel{e \in \mathcal{T}^{\prime}}{\longrightarrow} \stackrel{c \in \mathrm{S}}{\longrightarrow}\right)$ as $\stackrel{c^{\prime} \in \mathrm{S}}{\longrightarrow} \stackrel{\theta\left(d^{\prime}\right) \in \mathcal{T}^{\prime}}{\longrightarrow} . \gamma$ is well-defined because if $\theta\left(d_{1}\right)=\theta\left(d_{2}\right)$ then $E^{\prime}$ implies that $d_{1}=d_{2}$ and thus, by assumption $(\circ), \lambda\left(\stackrel{d_{1}}{\rightarrow} \stackrel{c}{\rightarrow}\right)=\lambda\left(\stackrel{d_{2}}{\rightarrow} \stackrel{c}{\rightarrow}\right)$.

Now, $\lambda, \gamma$ and $\theta$ satisfy the assumptions of Proposition 4.1|(i). In particular, (8) commutes by definition of $\gamma$ in terms of $\lambda$ and $\theta$. The conclusion of Proposition 4.1 guarantees that $\gamma$ is a distributive law. By construction, $\gamma$ is presented by the same equations as $\lambda$.

The proof of statement (ii) is analogous and uses Proposition 4.1.(ii). Finally, the same argument yields the two statements for PROPs in place of PROs.

We will use Proposition 4.2 in the next two sections (Theorem 5.1 and Lemma 6.5).

\subsection{Dual of a Distributive Law}

For later reference, we observe that, both for PROPs and PROs, a distributive law $\lambda: \mathcal{T} ; \mathrm{S} \rightarrow \mathrm{S} ; \mathcal{T}$ canonically induces one $\lambda^{\prime}: \mathrm{S}^{o p} ; \mathcal{T}^{o p} \rightarrow$ $\mathcal{T}^{o p} ; \mathrm{S}^{o p}$ defined as:

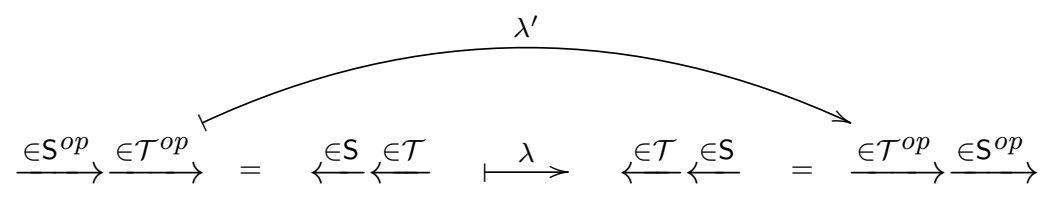

Proposition 4.3. $\lambda^{\prime}: \mathrm{S}^{o p} ; \mathcal{T}^{o p} \rightarrow \mathcal{T}^{o p} ; \mathrm{S}^{o p}$ is a distributive law of $P R O(P) s$. 
Proof. The main observation is that the unit and multiplication of $S^{o p}$ and of $\mathcal{T}^{o p}$ can be expressed in terms of those of $S$ and $\mathcal{T}$ in the same way as $\lambda^{\prime}$ is defined from $\lambda$. Then, using the fact that $\lambda$ is a distributive law, it is immediate to check that $\lambda^{\prime}$ makes diagrams $(6)$ commute.

\section{PROPs as Composed PROs}

In Example 3.3)(b) we saw that the PROP of monoids can be obtained from a distributive law $\mathrm{Mn} ; \mathrm{P} \rightarrow \mathrm{P}$; Mn of PROs. In this section we generalise this situation: any operadic monoidal presentation leads to a symmetric monoidal presentation by composition with the PRO of permutations. This is the conclusion of Theorem 5.1, the main result of this section.

Theorem 5.1. Let $(\Sigma, E)$ be a operadic monoidal presentation and $\mathrm{S}_{(\Sigma, E)}$ and $\mathcal{T}_{(\Sigma, E)}$ be its freely generated PRO and PROP respectively. Then there exists a distributive law $\mathrm{S}_{(\Sigma, E)} ; \mathrm{P} \rightarrow \mathrm{P} ; \mathrm{S}_{(\Sigma, E)}$ of PROs presented by equations

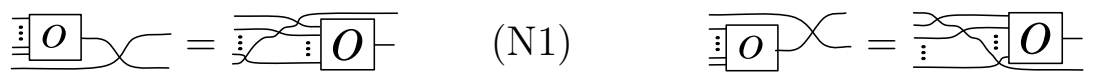

for each $o \in \Sigma$, such that $\mathrm{P} ; \mathrm{S}_{(\Sigma, E)}$ is a PROP. Moreover, there is an isomorphism of PROs $\mathcal{T}_{(\Sigma, E)} \cong \mathrm{P} ; \mathrm{S}_{(\Sigma, E)}$ that preserves the symmetry structure; i.e. it is also an isomorphism of PROPs.

Before we give the proof, note that the assumption that $(\Sigma, E)$ is operadic is crucial: e.g. if $\Sigma$ included an operation $o$ of coarity 2, there is no canonical way of putting $o ; D$ in the factorised form $\mathrm{P} ; \mathrm{S}_{(\Sigma, E)}$ : indeed the symmetry cannot, in general, be moved from the right to the left of $o$. Moreover, as we shall see in the proof below, the assumption that $E$ only involves terms of coarity 1 is important to ensure that it is respected by (N1)-(N2).

Proof. By construction, the $\operatorname{PROP} \mathcal{T}_{(\Sigma, E)}$ freely generated by $(\Sigma, E)$ is the same thing as the PRO $S_{\left(\Sigma^{\prime}, E^{\prime}\right)}$ freely generated by the signature $\Sigma^{\prime}:=$ $\Sigma \uplus\{\Phi\}$ and equations $E^{\prime}:=E \uplus\{(\mathrm{P} 1),(\mathrm{P} 2),(\mathrm{N} 1),(\mathrm{N} 2) \mid o \in \Sigma\}$. To see this, note that the addition of $D$ and equations $E^{\prime}$ amounts to a presentation of the laws of symmetric monoidal categories: besides the standard ( $(\mathrm{P} 1)$, equations $(\mathrm{P} 2),(\mathrm{N} 1)$ and $(\mathrm{N} 2)$ ensure that symmetries built from $\infty$ are natural. To prove the theorem, it thus suffices to show that:

$\mathrm{S}_{\left(\Sigma^{\prime}, E^{\prime}\right)}$ results from a distributive law of type

$$
\mathrm{S}_{(\Sigma, E)} ; \mathrm{P} \rightarrow \mathrm{P} ; \mathrm{S}_{(\Sigma, E)} \text { presented by (N1)-(N2). }
$$


In fact, we can further simplify our task, by throwing equations away. We claim that (†) follows from the following statement, where $\Upsilon=\{\Phi\}$ :

$\mathrm{S}_{\left.\left(\Sigma^{\prime},\{\mathbb{N} 1,, \mathbb{N 2}\}\right\}\right)}$ arises from a distributive law of type

$$
\mathrm{S}_{\Sigma} ; \mathrm{S}_{\Upsilon} \rightarrow \mathrm{S}_{\Upsilon} ; \mathrm{S}_{\Sigma} \text { presented by (N1)-(N2). }
$$

To see that $\$ \Rightarrow(\dagger)$, note that we can use Proposition 4.2 to lift a distributive law $\chi: \mathrm{S}_{\Sigma} ; \mathrm{S}_{\Upsilon} \rightarrow \mathrm{S}_{\Upsilon} ; \mathrm{S}_{\Sigma}$ to $\lambda: \mathrm{S}_{(\Sigma, E)} ; \mathrm{P} \rightarrow \mathrm{P} ; \mathrm{S}_{(\Sigma, E)}$ in two steps:

(a) first, if $\chi: S_{\Sigma} ; S_{\Upsilon} \rightarrow S_{\Upsilon} ; S_{\Sigma}$ is compatible with the equations (P1)(P2) of $\mathrm{P}$, we can "upgrade" it to $\gamma: \mathrm{S}_{\Sigma} ; \mathrm{P} \rightarrow \mathrm{P} ; \mathrm{S}_{\Sigma}$ by virtue of Proposition 4.2(ii),

(b) second, if $\gamma: \mathrm{S}_{\Sigma} ; \mathrm{P} \rightarrow \mathrm{P} ; \mathrm{S}_{\Sigma}$ is compatible with $E$, we can upgrade it to $\lambda: \mathrm{S}_{(\Sigma, E)} ; \mathrm{P} \rightarrow \mathrm{P} ; \mathrm{S}_{(\Sigma, E)}$, by virtue of Proposition 4.2 (i).

The two compatibility conditions above are easy to verify, using the assumption that $(\Sigma, E)$ is operadic. We give details in $\left(\mathrm{a}^{\prime}\right)$ and $\left(\mathrm{b}^{\prime}\right)$ below.

$\left(a^{\prime}\right)$ For (a), the assumption of Proposition 4.2)(ii) to be checked amounts to verify that, for any $\stackrel{c \in \mathrm{S}_{\Sigma}}{\longrightarrow}$ and naturals $n, m, \chi\left(\stackrel{c}{\rightarrow} \stackrel{i d_{n} \oplus l_{1} \oplus i d_{m}}{\longrightarrow}\right)=$ $\chi\left(\stackrel{c}{\rightarrow} \stackrel{i d_{n} \oplus r_{1} \oplus i d_{m}}{\rightarrow}\right)$ and $\chi\left(\stackrel{c}{\rightarrow} \stackrel{i d_{n} \oplus l_{2} \oplus i d_{m}}{\rightarrow}\right)=\chi\left(\stackrel{c}{\rightarrow} \stackrel{i d_{n} \oplus r_{2} \oplus i d_{m}}{\rightarrow}\right)$, where $l_{1}=$ $r_{1}$ and $l_{2}=r_{2}$ are the equations $(\mathrm{P} 1)$ and $(\mathrm{P} 2)$ respectively. Because $\Sigma$ is operadic, $c$ must be of shape $c_{1} \oplus \ldots \oplus c_{k}$, where each $c_{i}$ has coarity 1. Because any distributive law commutes with the monoidal product $\oplus$, our task reduces to check compatibility only for diagrams $c_{i}, c_{i+1}, c_{i+2}$ of coarity 1 that are connected to $l_{1}, r_{1}$ and to $l_{2}, r_{2}$, i.e. we need to verify that $\chi\left(\stackrel{c_{i} \oplus c_{i+1}}{\longrightarrow} \stackrel{l_{1}}{\rightarrow}\right)=\chi\left(\stackrel{c_{i} \oplus c_{i+1}}{\longrightarrow} \stackrel{r_{1}}{\longrightarrow}\right)$ and $\chi\left(\stackrel{c_{i} \oplus c_{i+1} \oplus c_{i+2}}{\longrightarrow} \stackrel{l_{2}}{\longrightarrow}\right.$ )$=\chi\left(\stackrel{c_{i} \oplus c_{i+1} \oplus c_{i+2}}{\longrightarrow} \stackrel{r_{2}}{\longrightarrow}\right)$. This is straightforward; for instance, for the equation $l_{1}=r_{1}$ we have:

$$
\begin{aligned}
& \left(c_{i} \oplus c_{i+1}\right) ; l_{1}=\stackrel{\overline{1} c_{i}}{\bar{\vdots} c_{i+1}} \longrightarrow \stackrel{\chi}{ } \\
& \text { P1)-P2] }
\end{aligned}
$$

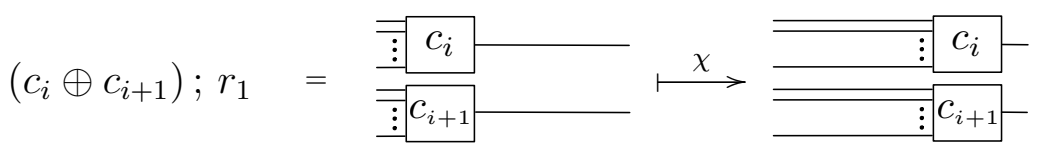


where $\chi$ has been computed by repeated application of the following consequences of (N1)-(N2), holding for any $\stackrel{c \in \mathrm{S}_{\Sigma}}{\longrightarrow}$ :

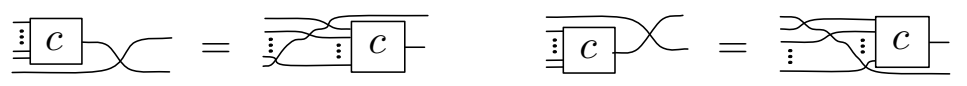

$\left(b^{\prime}\right)$ For (b), the assumption of Proposition 4.2) (i) to check is that, for any naturals $n, m$, permutation $\stackrel{p \in \mathrm{P}}{\longrightarrow}$ and equation $l=r$ in $E, \gamma\left(\stackrel{i d_{n} \oplus l \oplus i d_{m}}{\longrightarrow} \stackrel{p}{\longrightarrow}\right.$ )$=\gamma\left(\stackrel{i d_{n} \oplus r \oplus i d_{m}}{\rightarrow} \stackrel{p}{\rightarrow}\right)$. Observe that $l$ and $r$ both have coarity 1 , because $E$ is operadic. Now, by construction $\gamma$ is presented by the same equations (N1)-(N2) as $\chi$, thus the action of $\gamma$ on $\left(i d_{n} \oplus l \oplus i d_{m}\right) ; p$ and on $\left(i d_{n} \oplus r \oplus i d_{m}\right) ; p$ also amounts to a repeated application of (11). The key observation is that, in the process, instantiating $c$ to the subdiagram $l$ or to $r$ does not make any difference: they are treated as black boxes, connectivity being the only relevant information. It is thus evident that $\gamma\left(\stackrel{i d_{n} \oplus l \oplus i d_{m}}{\longrightarrow} \stackrel{p}{\rightarrow}\right)=\gamma\left(\stackrel{i d_{n} \oplus r \oplus i d_{m}}{\longrightarrow} \stackrel{p}{\rightarrow}\right)$.

So far, we proved that $(\varsubsetneqq) \Rightarrow(\uparrow)$; now we can conclude the proof of the main statement by showing $(\ddagger)$. As $S_{\left(\Sigma^{\prime},\{\sqrt{N 1}, \sqrt[N 2]{N 2}\}\right)}$ is the quotient of $S_{\Upsilon}+S_{\Sigma}$ by (N1) - N2), by Proposition 3.5 it suffices to construct a $\left(\mathrm{S}_{\Upsilon}, \mathrm{S}_{\Sigma}\right)$-factorisation system on $\mathrm{S}_{\left.\left(\Sigma^{\prime},\{\sqrt{\mathrm{N} 1}, \sqrt{\mathrm{N} 2}\}\right\}\right)}$. We define it via a terminating and convergent rewriting system on $S_{\left(\Sigma^{\prime},\{[\mathbb{N 1} 1, \sqrt[N 2]{N}\})\right.}$ (in the sense of Lafont [32]) transforming any diagram into a decomposed normal form $\stackrel{\in S_{\Upsilon}}{\longrightarrow} \stackrel{\in S_{\Sigma}}{\longrightarrow}$. The rewriting system is defined by taking as rules the equations (N1)-(N2), oriented from left to right, for each $o \in \Sigma$. Termination is witnessed by the following order: given a diagram $d$ of $S_{\left(\Sigma^{\prime},\left\{\left[\sqrt{N 1}, \sqrt{N_{2}}\right\}\right\}\right)}$, associate with each sub-diagram $=$, for some $o \in \Sigma$, the number of symmetries $\rightleftharpoons$ that are reachable from its output; the sum of these numbers is the value associated to $d$, which decreases at each application of a rewriting rule. For confluence, there is only one critical pair to check, for any two operations $o_{1}, o_{2} \in \Sigma$. For the sake of clarity, we show joinability below for $o_{1}, o_{2}$ of arity 2 , the generalisation to arbitrary arities 
being straightforward.

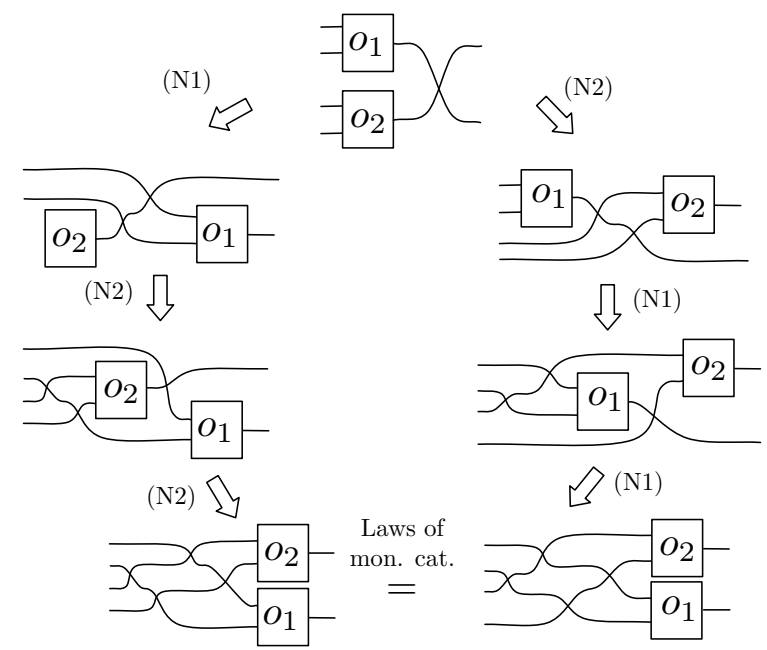

Termination and confluence yield uniqueness of the induced factorisation, hence by Proposition 3.5 a distributive law with the desired properties.

For later reference we state a useful corollary of Theorem 5.1, yielding a factorised form for the PROP CCm of commutative comonoids (Example 2.5 (c)). Note that the signature of $\mathrm{CCm}$ is not operadic. However, it is dual to an operadic signature, namely the one of commutative monoids.

Recall from Example 2.2 the PROs $\mathrm{Mn}$ and $\mathrm{Cm}$ freely generated by the monoidal presentations $\left(\Sigma_{M}, E_{M}\right)$ and $\left(\Sigma_{C}, E_{C}\right)$ of (non-commutative) monoids and comonoids respectively.

Proposition 5.2. There is a distributive law $\mathrm{P} ; \mathrm{Cm} \Rightarrow \mathrm{Cm} ; \mathrm{P}$ of PROs s.t. $\mathrm{Cm} ; \mathrm{P}$ is a PROP and $\mathrm{CCm}$ is isomorphic to its quotient by (A8).

Proof. As $\left(\Sigma_{M}, E_{M}\right)$ is operadic, Theorem 5.1 yields a distributive law $\lambda: \mathrm{Mn} ; \mathrm{P} \Rightarrow \mathrm{P} ; \mathrm{Mn}$ of PROs such that $\mathrm{P} ; \mathrm{Mn}$ is a PROP. Proposition 4.3 yields from $\lambda$ another distributive law $\gamma: \mathrm{P}^{o p} ; \mathrm{Mn}^{o p} \Rightarrow \mathrm{Mn}^{o p} ; \mathrm{P}^{o p}$. Using that, $\mathrm{Cm} \cong \mathrm{Mn}^{o p}$ and $\mathrm{P} \cong \mathrm{P}^{o p}, \gamma$ has in fact type $\mathrm{P} ; \mathrm{Cm} \Rightarrow \mathrm{Cm} ; \mathrm{P}$ and is presented by $(\mathrm{N} 1)^{o p}-(\mathrm{N} 2)^{o p}$, for $o \in\{\bullet, \bullet\}$, that means:

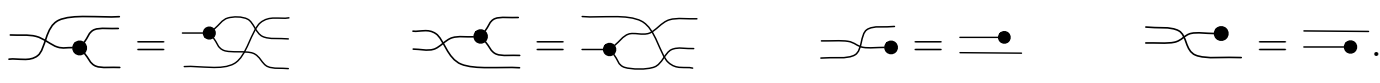

$\mathrm{Cm}$; $\mathrm{P}$ is a PROP: symmetries are natural because of $(\mathrm{N1})^{o p}-(\mathrm{N2})^{o p}$. Also, its quotient by $\mathrm{A} 8 \mathrm{~d}$ is by construction isomorphic to $\mathrm{C} \mathrm{Cm}$. 


\section{Lawvere Categories as Composed PROPs}

This section connects Lawvere categories and a certain class of distributive laws of PROPs. For a sketch of our approach, recall Example 2.8, describing the cartesian presentation of commutative monoids. First, note that the Lawvere category $\mathcal{L}_{\Sigma_{M}, E_{C M}}$ includes the PROP CMn freely generated by $\left(\Sigma_{M}, E_{C M}\right)$. Indeed, any string diagram of $\mathrm{CMn}$ can be interpreted as a list of terms following the recipe of Remark 2.9 . For instance,

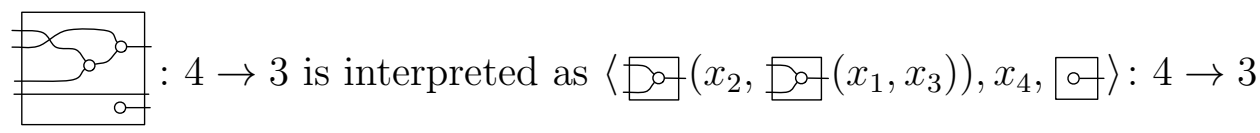

As we observed above, string diagrams of $\mathrm{CMn}$ express linear terms. What makes $\mathcal{L}_{\Sigma_{M}, E_{M}}$ more general is the ability to copy and discard variables. Indeed, as in any monoidal category where $\oplus$ is the cartesian product, $\mathcal{L}_{\Sigma_{M}, E_{M}}$ comes equipped with a canonical choice of "copy" and "discard" operations $\operatorname{cpy}(n):=\left\langle x_{1}, \ldots, x_{n}, x_{1}, \ldots, x_{n}\right\rangle: n \rightarrow 2 n \quad d s c(n):=\langle\rangle: n \rightarrow 0 \quad n \in \mathbb{N}$

natural in $n$, which satisfy the expected equations: copying is commutative and associative; copying then discarding is the same as not doing anything - see e.g. [23, 18].

How can we mimic the copy and discard structure in the language of PROPs? First, for each $n>1$ one can define $\operatorname{cpy}(n)$ and $d s c(n)$ in terms of $\operatorname{cpy}(1)$ and $d s c(1)$, which can therefore be regarded as the only fundamental operations [ $^{6}$ Also, the equations that they satisfy amount to saying that cpy(1) is the comultiplication and $d s c(1)$ the counit of a commutative comonoid on 1. Therefore, they are the generators of the PROP CCm:

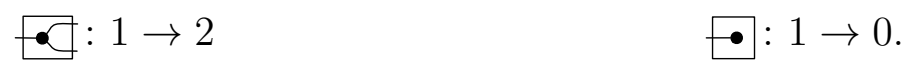

Following this line of thought, it is clear that $\mathrm{CMn}$ and $\mathrm{CCm}$ "live" inside $\mathcal{L}_{\Sigma_{M}, E_{C M}}$. We claim that these two PROPs provide a complete description of $\mathcal{L}_{\Sigma_{M}, E_{C M}}$, that means, any arrow of $\mathcal{L}_{\Sigma_{M}, E_{C M}}$ can be presented diagrammatically as an arrow of $\mathrm{CCm}$ followed by an arrow of $\mathrm{CMn}$. For instance:

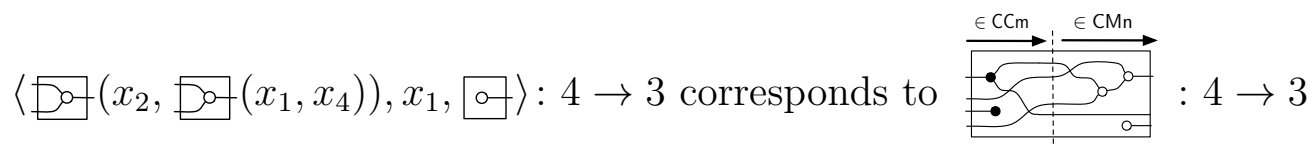

\footnotetext{
${ }^{6}$ For $n=0$, both operations are equal to the identity on 0 .
} 
Observe that the diagram is of the form $\stackrel{\in \mathrm{CCm}}{\longrightarrow} \stackrel{\in \mathrm{CMn}}{\longrightarrow}$. Intuitively, $\mathrm{CCm}$ is deputed to capture the interplay of variables - in this case, the fact that $x_{1}$ is copied and $x_{3}$ is unused (discarded) - and CMn describes the syntactic tree of the terms. Of course, for this factorisation to be possible in general, we need additional equations to account for the composition of diagrams. For instance:

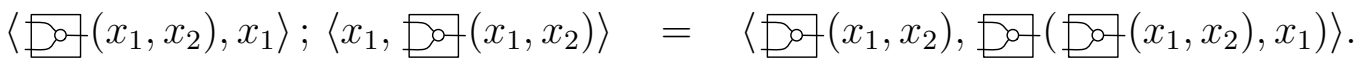

$$
\begin{aligned}
& \text {; } \quad \stackrel{?}{=}
\end{aligned}
$$

The second equality holds if we use equation $\mathrm{A} 10$ of the SMP of bialgebras (Example 2.5(d)). Thus the example suggests that composition by substitution in $\mathcal{L}_{\Sigma_{M}, E_{M}}$ can be seen at the diagrammatic level through the use of bialgebra equations, which as we know from Example 3.4 (b) present the composite PROP CCm; CMn, defined by the "pullback" distributive law. Thus, it would seem that $\mathcal{L}_{\Sigma_{M}, E_{C M}} \cong \mathrm{CCm} ; \mathrm{CMn}$, i.e. the Lawvere category of commutative monoids is isomorphic, as a PROP, to the PROP of commutative bialgebras. This is indeed the case, and as we shall show below.

Theorem 6.1. Suppose $(\Sigma, E)$ is a cartesian $S M P$ and $\mathcal{T}_{(\Sigma, E)}$ is its freely generated PROP. Then we have an isomorphism of PROPs $\mathcal{L}_{(\Sigma, E)} \cong \mathrm{CCm} ; \mathcal{T}_{(\Sigma, E)}$, where $\mathcal{T}_{(\Sigma, E)} ; \mathrm{CCm} \rightarrow \mathrm{CCm} ; \mathcal{T}_{(\Sigma, E)}$ is the distributive law presented by the following equations, for each $o \in \Sigma$ :

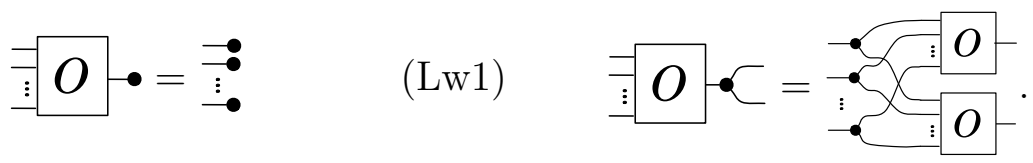

Before moving to the proof of Theorem 6.1, we show its significance by revisiting some well-known presentations in terms of our result.

\section{Example 6.2.}

(a) Theorem 6.1 instantiated on the SMP CMn of commutative monoids (Example 2.5 (b) results with (Lw1)-(Lw2) being the bialgebra equations (A9)-(A12). The isomorphism $\mathrm{B} \cong \mathrm{CCm} ; \mathrm{CMn}$ (Example 3.4.(b)) is an immediate consequence and thus the Lawvere category of commutative monoids - seen as a PROP_is the PROP of bialgebras. 
(b) As forecast by Remark 2.9, not every Lawvere category is a composite. For instance, the cartesian presentation $\left(\Sigma_{G}, E_{G}\right)$ of abelian groups extends the one $\left(\Sigma_{M}, E_{M}\right)$ of commutative monoids with an inverse operation $\square: 1 \rightarrow 1$ and a non-linear equation $\mathrm{D}_{\mathrm{O}}(x, \mathbf{\square}(x))=0$. Here Theorem 6.1 still yields useful information about the structure of the resulting Lawvere category. For instance, it means that $\mathcal{L}_{\left(\Sigma_{G}, E_{G}\right)}$, seen as a PROP, is isomorphic to the PROP $\mathrm{CCm} ; \mathcal{T}_{\Sigma_{G}, E_{M}}$ quotiented by the above non-linear equation, which rendered as string diagrams is:

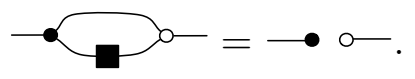

Interestingly, the result of this quotient is isomorphic to the PROP of integer matrices, see e.g. [12] and its models in a symmetric monoidal category are the Hopf algebras [22], with $\square$ the antipode.

(c) In [26] Fritz presents the category of finite sets and probabilistic maps using generators and equations. The resulting Lawvere category $\mathcal{L}_{\text {Prob }}$ can be decomposed following the scheme of Theorem 6.1: there is a linear part $\left(\Sigma_{P}, E_{P}\right)$ of the presentation - given by binary convex combinations $\Sigma_{P}=\{-\lambda-: 2 \rightarrow 1 \mid \lambda \in[0,1]\}$ and suitable associativity and commutativity laws in $E_{P}$, a commutative comonoid structure, and the two interact according to (Lw1)-(Lw2). This interaction yields a composite PROP CCm; $\mathcal{T}_{\left(\Sigma_{P}, E_{P}\right)}$ which, quotiented by non-linear equations $\exists 0-=\longrightarrow$ and $\cdot\left[\lambda-=-\right.$, yields $\mathcal{L}_{\text {Prob }} \cdot 7$

Remark 6.3. It is instructive examine the ramifications of Theorem 6.1 for models. Let $(\Sigma, E)$ be as in the statement of the theorem. We recalled models

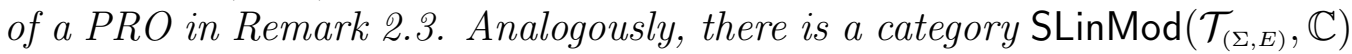
of models of the PROP $\mathcal{T}_{(\Sigma, E)}$ in a symmetric strict monoidal category $\mathbb{C}$, with objects given by symmetric strict monoidal functors $\mathcal{T}_{(\Sigma, E)} \rightarrow \mathbb{C}$.

For $\mathbb{C}$ cartesian, models of the Lawvere category $\mathcal{L}_{(\Sigma, E)}$ are cartesian (i.e., finite-products preserving) functors $\mathcal{L}_{(\Sigma, E)} \rightarrow \mathbb{C}$, forming a category $\operatorname{CartMod}\left(\mathcal{L}_{(\Sigma, E)}, \mathbb{C}\right)$. Now, we have that models of $\mathcal{L}_{(\Sigma, E)}$ in $\mathbb{C}$ cartesian are

\footnotetext{
${ }^{7}$ In fact, the Lawvere category in [26] has finite coproducts, while our $\mathcal{L}_{\text {Prob }}$ is based on finite products. This is just a matter of co-/contra-variant presentation of the same data: one can switch between the two by "mirroring" diagrams.
} 
the same as models of $\mathcal{T}_{(\Sigma, E)}$ in $\mathbb{C}$, seen as a symmetric strict monoidal category. Indeed, there are equivalences:

$$
\operatorname{LinMod}\left(\mathcal{T}_{(\Sigma, E)}, \mathbb{C}\right) \simeq \operatorname{LinMod}\left(\operatorname{CCm} ; \mathcal{T}_{(\Sigma, E)}, \mathbb{C}\right) \simeq \operatorname{CartMod}\left(\mathcal{L}_{(\Sigma, E)}, \mathbb{C}\right)
$$

The reason for the second equivalence is that any cartesian category carries exactly one commutative comonoid structure: to see this, note that given two comonoid structures, the counits must agree since 0 is terminal. From this, it is easy to show that the comultiplications must agree as well. Thus a symmetric monoidal functor from $\mathrm{CCm} ; \mathcal{T}_{(\Sigma, E)}$ to cartesian $\mathbb{C}$ is obliged to take the comonoid structure of $\mathrm{C} C \mathrm{~m}$ to that induced by the diagonals in $\mathbb{C}$. The first equivalence, instead, is induced by the naturality of the diagonals in $\mathbb{C}$ : roughly speaking, the additional equations imposed by the distributive law defining $\mathrm{CCm} ; \mathcal{T}_{(\Sigma, E)}$ are imposed implicitly by the cartesian structure of $\mathbb{C}$.

The remainder of the section is devoted to proving Theorem 6.1. A first, useful observation is that in $\mathcal{T}_{\Sigma}$, the free PROP on $(\Sigma, \emptyset)$, (Lw1)-(Lw2) allow us to copy and discard not only the generators but arbitrary string diagrams.

Lemma 6.4. Suppose $\bar{d}$ is an arrow of $\mathcal{T}_{\Sigma}$. Then the following holds in $\mathcal{T}_{\Sigma}+\mathrm{CCm}$ quotiented by (Lw1)-(Lw2).
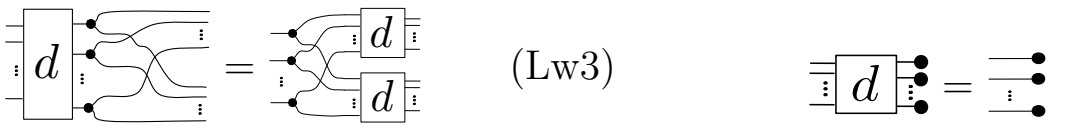

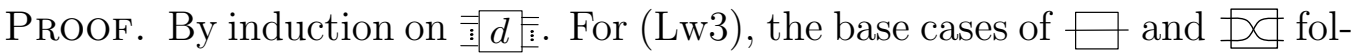
low by the laws of SMCs (Figure 2). The base case of $\overline{\mathrm{i}}-$, for $o$ a generator in $\Sigma$, is given by (Lw2). The inductive cases of ; and $\oplus$ follow immediately by the induction hypothesis. The reasoning for ( $\mathrm{Lw} 4$ is analogous.

Next, we observe that it suffices to prove Theorem 6.1 for SMPs with no equations, as $\mathcal{T}_{\Sigma}$ above. With this insight, computations in $\mathcal{L}_{(\Sigma, E)}$ are simpler, since we can work with terms instead of equivalence classes.

Lemma 6.5. If the statement of Theorem 6.1 holds in the case $E=\varnothing$, then it holds for any cartesian $\operatorname{SMP}(\Sigma, E)$. 
Proof. Let $(\Sigma, E)$ be a cartesian SMP and $\mathcal{T}_{\Sigma}, \mathcal{T}_{(\Sigma, E)}$ be PROPs freely generated, respectively, by $(\Sigma, \emptyset)$ and $(\Sigma, E)$. By assumption, Theorem 6.1 holds for $(\Sigma, \emptyset)$ with distributive law $\lambda: \mathcal{T}_{\Sigma} ; \mathrm{CCm} \rightarrow \mathrm{CCm} ; \mathcal{T}_{\Sigma}$. We now want to show that $\lambda$ is compatible with $E$, in the sense of Proposition 4.2 [i); it suffices to prove that $\lambda\left(\stackrel{i d_{n} \oplus l \oplus i d_{m} \in \mathcal{T}_{\Sigma}}{\longrightarrow} \stackrel{d \in \mathrm{CCm}}{\longrightarrow}\right)=\lambda\left(\stackrel{i d_{n} \oplus r \oplus i d_{m} \in \mathcal{T}_{\Sigma}}{\longrightarrow} \stackrel{d \in \mathrm{CCm}}{\longrightarrow}\right)$ for all $d, n, m$ and equations $l=r$ in $E$. Now, as a first step, we can apply Proposition 5.2 to decompose $\stackrel{d \in \mathrm{CCm}}{\longrightarrow}$ as $\stackrel{d^{\prime} \in \mathrm{Cm}}{\longrightarrow} \stackrel{p \in \mathrm{P}}{\longrightarrow}$, where $\mathrm{Cm}$ is the free PRO of comonoids (Example 2.2( (c)). We shall interpret $d^{\prime}$ as an arrow of $\mathrm{CCm}$ : this is harmless since the terms yielding the arrows of $\mathrm{Cm}$ are a subset of those generating the arrows of $\mathrm{CCm}$. We thus have

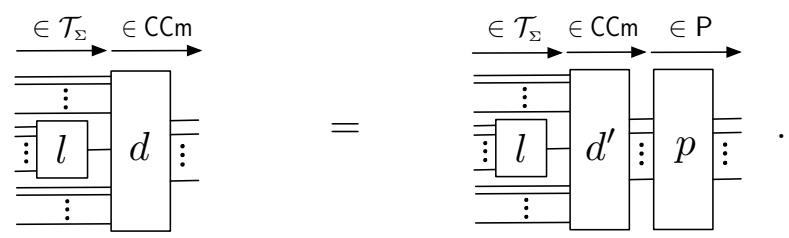

Note that, because $E$ is operadic, $l$ and $r$ have coarity 1 . Using the fact that $d^{\prime}$ does not contain symmetries, we can further decompose it as follows.

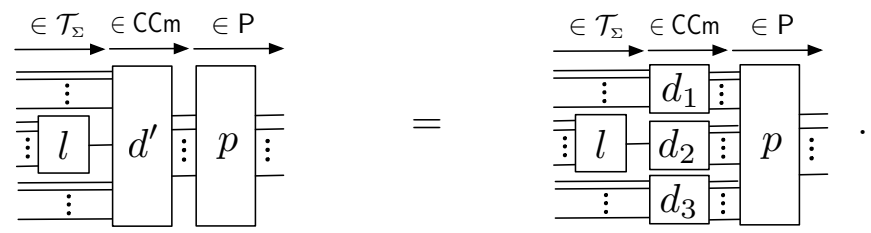

We now exploit the fact that distributive laws of PROPs preserve the symmetric monoidal structure. That means, $\lambda\left(\stackrel{i d_{n} \oplus l \oplus i d_{m}}{\longrightarrow} \stackrel{d^{\prime} ; p}{\longrightarrow}\right)$ can be computed componentwise as follows (observe that $\stackrel{p \in \mathrm{P}}{\longrightarrow}$ qualifies both as an arrow of $\mathcal{T}_{\Sigma}$ and of $\mathrm{CCm}$, as any PROP contains a copy of P, cf. Example 2.2 (a)).
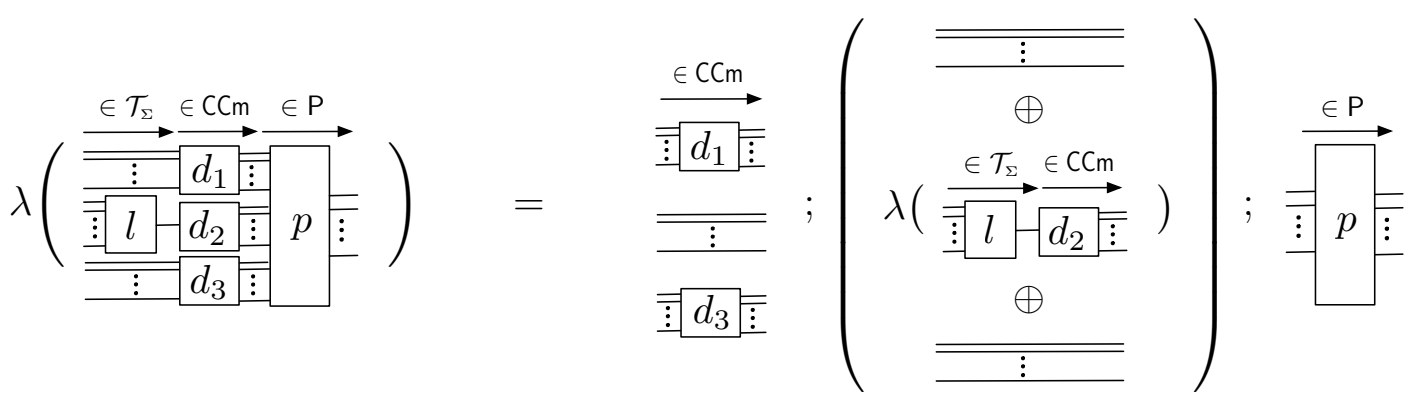
To conclude, we need to ensure that $\lambda\left(\stackrel{l}{\rightarrow} \stackrel{d_{2}}{\longrightarrow}\right)=\lambda\left(\stackrel{r}{\rightarrow} \stackrel{d_{2}}{\longrightarrow}\right)$. This is obvious once we realise that, because $d_{2}$ has arity 1 , it can be only of two shapes, yielding the cases below. The value of $\lambda$ is computed using (Lw3)-(Lw4).

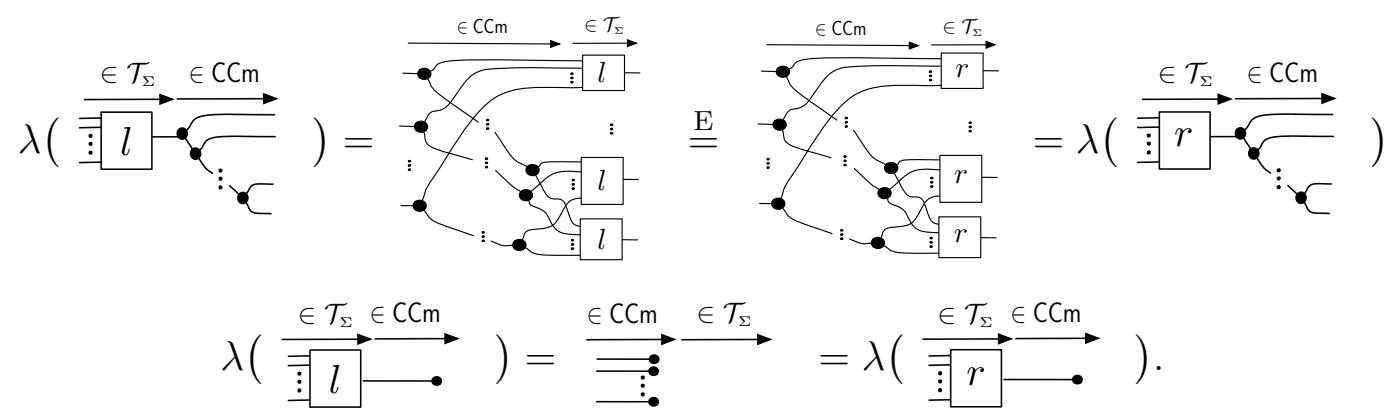

Therefore, we verified the assumption of Proposition 4.2(i), which yields distributive law $\lambda^{\prime}: \mathcal{T}_{(\Sigma, E)} ; \mathrm{CCm} \rightarrow \mathrm{CCm} ; \mathcal{T}_{(\Sigma, E)}$ as desired.

Thus, to prove Theorem 6.1, it suffices to focus on $\mathcal{L}_{\Sigma}$ (an abbreviation for $\left.\mathcal{L}_{\Sigma, \emptyset}\right)$. We shall obtain the distributive law $\mathcal{T}_{\Sigma} ; \mathrm{CCm} \rightarrow \mathrm{CCm} ; \mathcal{T}_{\Sigma}$ using Proposition 3.6, by showing that any arrow of $\mathcal{L}_{\Sigma}$ decomposes as

$$
\stackrel{\in \mathrm{CCm}}{\longrightarrow} \stackrel{\in \mathcal{T}_{\Sigma}}{\longrightarrow}
$$

Note that both $\mathcal{T}_{\Sigma}$ and $\mathrm{CCm}$ can be seen as sub-PROPs of $\mathcal{L}_{\Sigma}$ :

\section{Lemma 6.6.}

- $\mathrm{CCm}$ is the sub-PROP of $\mathcal{L}_{\Sigma}$ whose arrows are lists of variables. The inclusion $\Phi: \mathrm{CCm} \rightarrow \mathcal{L}_{\Sigma}$ is defined on generators of $\mathrm{CCm}$ as follows:

$$
\bullet \mapsto\left\langle x_{1}, x_{1}\right\rangle: 1 \rightarrow 2 \quad \leftarrow \mapsto\langle\rangle: 1 \rightarrow 0
$$

- $\mathcal{T}_{\Sigma}$ is the sub-PROP of $\mathcal{L}_{\Sigma}$ whose arrows are linear terms. The inclusion $\Psi: \mathcal{T}_{\Sigma} \rightarrow \mathcal{L}_{\Sigma}$ is defined on generators of $\mathcal{T}_{\Sigma}$ by:

$$
\overline{\overline{\mathrm{i}}} \boldsymbol{O}-\mapsto\left\langle o\left(x_{1}, \ldots, x_{n}\right)\right\rangle: n \rightarrow 1 \quad(o: n \rightarrow 1) \in \Sigma .
$$

Proof. $C C m \cong F^{o p}$ is the initial Lawvere category (free category with products on one object) and can clearly be identified with the "variable-only" fragment of $\mathcal{L}_{\Sigma}$. One can easily verify that $\Psi: \mathcal{T}_{\Sigma} \rightarrow \mathcal{L}_{\Sigma}$ identifies the linear terms in $\mathcal{L}_{\Sigma}$ following the observations in Remark 2.9 . 
Henceforth we shall not distinguish between $\mathrm{CCm}$ and the isomorphic sub-PROP of $\mathcal{L}_{\Sigma}$ identified by the image of $\Phi$, and similarly for $\mathcal{T}_{\Sigma}$ and $\Psi$. Lemma 6.6 allows us to see $\mathcal{L}_{\Sigma}$ as a playground where $\mathrm{CCm}$ and $\mathcal{T}_{\Sigma}$ interact. The following statement guarantees the soundness of the interaction described by (Lw1)-(Lw2). Indeed, it is immediate that:

Lemma 6.7. (Lw1) and (Lw2) are sound in $\mathcal{L}_{\Sigma}$.

We now have all the ingredients to conclude the proof of Theorem 6.1.

Proof (Theorem 6.1). The conclusion of Lemma 6.5 allows us to assume, without loss of generality, that $E=\varnothing$. This means that, in $\mathcal{L}_{\Sigma}$, any arrow $n \stackrel{f}{\rightarrow} m$ can easily be factorised as $n \stackrel{\hat{c} \in \mathrm{CCm}}{\longrightarrow} \stackrel{\hat{d} \in \mathcal{T}_{\Sigma}}{\longrightarrow} m$.

Indeed, $f$ is just a list of cartesian $\Sigma$-terms $\left\langle t_{1}, \ldots, t_{m}\right\rangle$. Replacing the variables of $\left\langle t_{1}, \ldots, t_{m}\right\rangle$ with fresh ones $x_{1}, \ldots, x_{z}$, without repetition, gives us a list of linear terms $z \stackrel{\hat{d} \in \mathcal{T}_{\Sigma}}{\longrightarrow} m$. Component $\hat{c}$ is the list $n \stackrel{\text { ovar }\left(t_{1}, \ldots, t_{m}\right) \in \mathrm{CCm}}{\longrightarrow} z$ of variables originally occurring in $f$, so that post-composition with $\hat{d}$ yields $\left\langle t_{1}, \ldots, t_{m}\right\rangle$. Uniqueness up-to permutation of this factorisation is immediate.

Therefore, Proposition 3.6 gives us a distributive law $\lambda: \mathcal{T}_{\Sigma} ; \mathrm{CCm} \rightarrow$ $\mathrm{CCm} ; \mathcal{T}_{\Sigma}$ and $\mathcal{L}_{\Sigma} \cong \mathrm{CCm} ; \mathcal{T}_{\Sigma}$. It remains to show that (Lw1)-(Lw2) suffice to derive all equations implied by $\lambda$. Suppose, therefore, that $\lambda(d ; c)=c^{\prime} ; d^{\prime}$. We need to show that:

$$
d ; c=c^{\prime} ; d^{\prime} \text { modulo the equations of } \mathcal{T}_{\Sigma}+\mathrm{CCm} \text { and }(\mathrm{Lw} 1)-(\mathrm{Lw} 2) .
$$

Since the factorisation is unique up-to permutation, it suffices to show that

there exists factorisation $n \stackrel{c^{\prime \prime} \in \mathrm{CCm}}{\longrightarrow} \stackrel{d^{\prime \prime} \in \mathcal{T}_{\Sigma}}{\longrightarrow} m$ of $d ; c$ in $\mathcal{L}_{\Sigma}$ s.t. $d ; c$ and $c^{\prime \prime} ; d^{\prime \prime}$ are equal modulo the equations of $\mathcal{T}_{\Sigma}+\mathrm{CCm}$ and $(\mathrm{Lw} 1)-(\mathrm{Lw} 2)$.

Statement $(\ddagger)$ implies $(\dagger)$ because, by uniqueness up-to permutation, there exists $\stackrel{p \in \mathrm{P}}{\longrightarrow}$ such that $d^{\prime}=p ; d^{\prime \prime}$ and $c^{\prime \prime}=c^{\prime} ; p$ in $\mathcal{L}_{\Sigma}$. Since $p$ is an arrow of both sub-PROPs $\mathcal{T}_{\Sigma}$ and $\mathrm{CCm}$, the first equality also holds in $\mathcal{T}_{\Sigma}$ and the second in $\mathrm{CCm}$. So $c^{\prime} ; d^{\prime}=c^{\prime} ; p ; d^{\prime \prime}=c^{\prime \prime} ; d^{\prime \prime}$ in $\mathcal{T}_{\Sigma}+\mathrm{CCm}$.

To prove $(\ddagger)$, we give a procedure to transform $\stackrel{d \in \mathcal{T}_{\Sigma}}{\longrightarrow} \stackrel{c \in \mathrm{CCm}}{\longrightarrow}$ into the form $\stackrel{c^{\prime \prime} \in \mathrm{CCm}}{\longrightarrow} \stackrel{d^{\prime \prime} \in \mathcal{T}_{\Sigma}}{\longrightarrow}$ by only using the equations in $\mathcal{T}_{\Sigma}+\mathrm{CCm}$ plus (Lw1)-(Lw2). Lemmas 6.6 and 6.7 guarantee that $d ; c=c^{\prime \prime} ; d^{\prime \prime}$ as arrows of $\mathcal{L}_{\Sigma}$. 
1. Recall that Theorem 5.1 yields that $\mathcal{T}_{\Sigma} \cong \mathrm{P} ; \mathrm{S}_{\Sigma}$, where $\mathrm{S}_{\Sigma}$ is the free PRO on $\Sigma$. Thus $\stackrel{d \in \mathcal{T}_{\Sigma}}{\longrightarrow}$ can be decomposed as $\stackrel{p \in \mathrm{P}}{\longrightarrow} ; \stackrel{\bar{d} \in \mathrm{S}_{\Sigma}}{\longrightarrow}$.

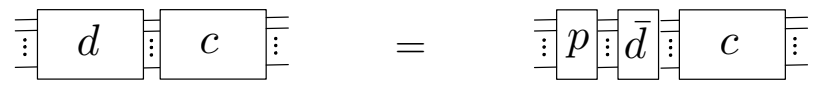

Similarly, by Proposition $5.2, c=\stackrel{\bar{c} \in \mathrm{Cm}}{\longrightarrow} \stackrel{p^{\prime} \in \mathrm{P}}{\longrightarrow}$, where $\mathrm{Cm}$ is the free PRO of comonoids.

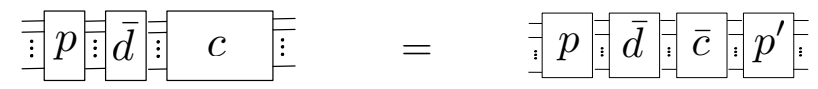

By doing the above, we moved the permutations out of the way.

2. We now make $\bar{d}$ and $\bar{c}$ interact. First note that, since $\bar{d}$ comes from $\mathrm{S}_{\Sigma}$, it does not contain $\Phi$ and all generators $o \in \Sigma$ have coarity 1. Thus $\bar{d}$ must the $\oplus$-product $\bar{d}_{1} \oplus \ldots \oplus \bar{d}_{z}$ of string diagrams $\bar{d}_{i}: k_{i} \rightarrow 1$ of $\mathcal{T}_{\Sigma}$.

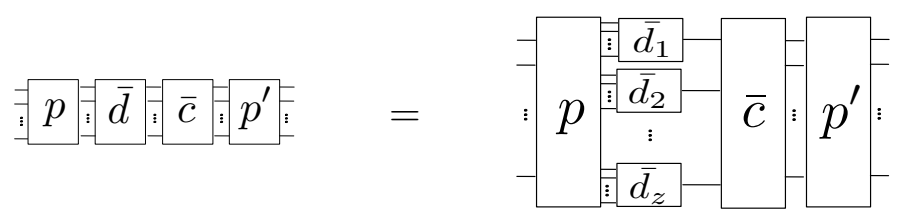

For analogous reasons, $\bar{c}$ is also a $\oplus$-product $\overline{c_{1}} \oplus \ldots \oplus \overline{c_{z}}$ where, for $1 \leq i \leq z$

$$
-\overline{\bar{c}_{i} \bar{i}}=\sqrt{C_{i}} \quad \text { or } \quad-\overline{c_{i}}=\cdots
$$

We thus can present $\bar{c}$ as follows:

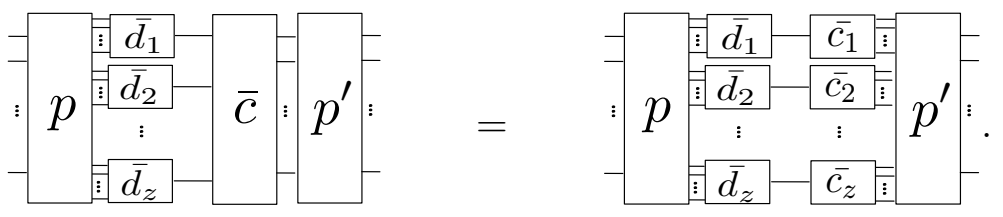

We are now in position to distribute each $\bar{d}_{i}$ over the corresponding $\bar{c}_{i}$. Suppose first $\bar{c}_{i}$ satisfies the left-hand equality in (12). By assumption, all the equations of $\mathcal{T}_{\Sigma}+\mathrm{CCm}$, (Lw1) and (Lw2 hold. Thus, by 
Lemma 6.4, also Lw3 holds. Starting from $\bar{d}_{i} ; \bar{c}_{i}$, we can iteratively apply (Lw3) to obtain an arrow of the form $\stackrel{\in \mathrm{CCm}}{\longrightarrow} \stackrel{\in \mathcal{T}_{\Sigma}}{\longrightarrow}$.

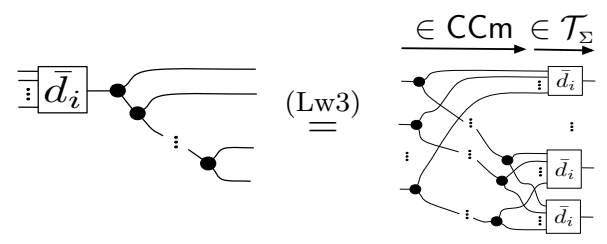

In the remaining case, $\bar{c}_{i}$ satisfies the right-hand equality in $(12)$. Then, one application of (Lw4) also gives us a string diagram of shape $\stackrel{\in \mathrm{CCm}}{\longrightarrow} \stackrel{\in \mathcal{T}_{\Sigma}}{\longrightarrow}$.

$$
\bar{i} \overline{\bar{d}_{i}} \cdot \stackrel{\mathrm{Lw} 4}{=} \stackrel{\in \mathrm{CCm} \in \mathcal{T}_{\Sigma}}{\longrightarrow}
$$

Applying the above transformations for each $\bar{d}_{i} ; \bar{c}_{i}$ yields a string diagram of the desired shape $\stackrel{c^{\prime \prime} \in \mathrm{CCm}}{\longrightarrow} \stackrel{d^{\prime \prime} \in \mathcal{T}_{\Sigma}}{\longrightarrow}$.

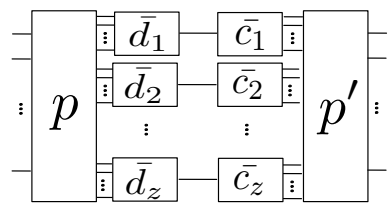

Lw3, Lw4

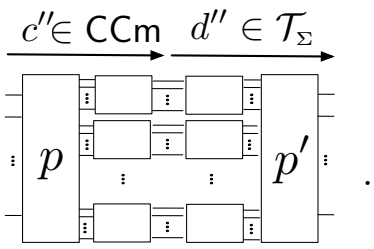

Observe that the transformations above use only equations in $\mathcal{T}_{\Sigma}+\mathrm{CCm}$, (Lw1) and (Lw2). This concludes the proof of $(\ddagger)$, and thus of the theorem.

Acknowledgements. The first author acknowledge support by project ANR 12IS02001 PACE. The third author acknowledges support from the European Research Council under the European Union's Seventh Framework Programme (FP7/2007-2013) / ERC grant agreement no 320571.

\section{References}

[1] Baez, J., Erbele, J.: Categories in control. Theory and Application of Categories 30, 836-881 (2015)

[2] Baez, J.C., Stay, M.: Physics, topology, logic and computation: A rosetta stone. Lecture Notes in Physics 813, 95-174 (2011) 
[3] Baez, J.C.: Universal algebra and diagrammatic reasoning. Lecture (2006), http://math.ucr.edu/home/baez/universal/universal_ hyper.pdf

[4] Bénabou, J.: Introduction to bicategories. In: Reports of the Midwest Category Seminar, Lecture Notes in Mathematics, vol. 47, pp. 1-77. Springer Berlin Heidelberg (1967)

[5] Blackwell, R., Kelly, G., Power, A.: Two-dimensional monad theory. Journal of Pure and Applied Algebra 59(1), 1 - 41 (1989), http://www . sciencedirect.com/science/article/pii/0022404989901606

[6] Bonchi, F., Milius, S., Silva, A., Zanasi, F.: Killing epsilons with a dagger - a coalgebraic study of systems with algebraic label structure. Theoretical Computer Science 604, 102-126 (2015)

[7] Bonchi, F., Sobocinski, P., Zanasi, F.: A categorical semantics of signal flow graphs. In: CONCUR 2014 - Concurrency Theory - 25th International Conference, CONCUR 2014. Proceedings. pp. 435-450 (2014)

[8] Bonchi, F., Sobocinski, P., Zanasi, F.: Interacting bialgebras are Frobenius. In: Foundations of Software Science and Computation Structures 17th International Conference, FOSSACS 2014, Held as Part of the European Joint Conferences on Theory and Practice of Software, ETAPS 2014. pp. 351-365 (2014)

[9] Bonchi, F., Sobocinski, P., Zanasi, F.: Full abstraction for signal flow graphs. In: Proceedings of the 42nd Annual ACM SIGPLAN-SIGACT Symposium on Principles of Programming Languages, POPL 2015. pp. $515-526(2015)$

[10] Bonchi, F., Sobocinski, P., Zanasi, F.: Lawvere categories as composed props. In: Coalgebraic Methods in Computer Science - 13th IFIP WG 1.3 International Workshop, CMCS 2016, Colocated with ETAPS 2016, Eindhoven, The Netherlands, April 2-3, 2016, Revised Selected Papers. Lecture Notes in Computer Science, vol. 9608, pp. 11-32. Springer (2016), http://dx.doi.org/10.1007/978-3-319-40370-0_3

[11] Bonchi, F., Sobocinski, P., Zanasi, F.: The calculus of signal flow diagrams I: linear relations on streams. Inf. Comput. 252, 2-29 (2017) 
[12] Bonchi, F., Sobociński, P., Zanasi, F.: Interacting Hopf algebras. Journal of Pure and Applied Algebra 221(1), 144-184 (January 2017)

[13] Bonchi, F., Zanasi, F.: Bialgebraic semantics for logic programming. Logical Methods in Computer Science 11(1:14) (2015)

[14] Bonsangue, M.M., Hansen, H.H., Kurz, A., Rot, J.: Presenting distributive laws. In: Algebra and Coalgebra in Computer Science - 5th International Conference, CALCO 2013. Proceedings. pp. 95-109 (2013)

[15] Borceux, F.: Handbook of Categorical Algebra 1 - Basic Category Theory. Cambridge Univ. Press (1994)

[16] Bruni, R., Melgratti, H.C., Montanari, U.: A connector algebra for P/T nets interactions. In: CONCUR '11. pp. 312-326. Springer (2011)

[17] Bruni, R., Montanari, U., Plotkin, G.D., Terreni, D.: On hierarchical graphs: Reconciling bigraphs, gs-monoidal theories and gs-graphs. Fundam. Inform. 134(3-4), 287-317 (2014)

[18] Burroni, A.: Higher dimensional word problems with applications to equational logic. Theor Comput Sci 115, 43-62 (1993)

[19] Cheng, E.: Distributive laws for lawvere theories. Algebra Universalis (to appear), available at http://arxiv.org/abs/1112.3076

[20] Coecke, B., Duncan, R.: Interacting quantum observables: categorical algebra and diagrammatics. New Journal of Physics 13(4), 043016 (2011)

[21] Corradini, A., Gadducci, F.: An algebraic presentation of term graphs, via gs-monoidal categories. Applied Categorical Structures 7(4), 299-331 (1999)

[22] Dascalescu, S., Nastasescu, C., Raianu, S.: Hopf Algebras, Pure and Applied Mathematics, vol. 235. Marcel Dekker, Inc., New York, Basel (2001), an Introduction

[23] Eilenberg, S., Kelly, G.M.: Closed categories. In: Proceedings of the Conference on Categorical Algebra. pp. 421-562. Springer (1966)

[24] Fiore, M.P., Campos, M.D.: The algebra of directed acyclic graphs. In: Abramsky Festschrift. LNCS, vol. 7860 (2013) 
[25] Fong, B., Rapisarda, P., Sobociński, P.: A categorical approach to open \& interconnected dynamical systems pp. 495-504 (2016)

[26] Fritz, T.: A presentation of the category of stochastic matrices. CoRR abs/0902.2554 (2009), http://arxiv.org/abs/0902.2554

[27] Hyland, M., Plotkin, G., Power, J.: Combining effects: sum and tensor. Theor Comput Sci 357(1-3), 70-99 (2006)

[28] Hyland, M., Power, J.: Symmetric monoidal sketches. In: PPDP. pp. 280-288 (2000)

[29] Hyland, M., Power, J.: The category theoretic understanding of universal algebra: Lawvere theories and monads. Electronic Notes in Theoretical Computer Science 172, 437-458 (2007)

[30] Jacobs, B.: Semantics of weakening and contraction. Annals of Pure and Applied Logic 69(1), 73 - 106 (1994)

[31] Lack, S.: Composing PROPs. Theor App Categories 13(9), 147-163 (2004)

[32] Lafont, Y.: Equational reasoning with 2-dimensional diagrams. In: Comon, H., Jounnaud, J.P. (eds.) Term Rewriting, Lecture Notes in Computer Science, vol. 909, pp. 170-195. Springer Berlin Heidelberg (1995)

[33] Lawvere, W.F.: Functorial Semantics of Algebraic Theories. Ph.D. thesis (2004)

[34] Mac Lane, S.: Categorical algebra. B Am Math Soc 71, 40-106 (1965)

[35] Mac Lane, S.: Categories for the Working Mathematician. Springer (1998)

[36] Melliès, P.A.: Categorical semantics of linear logic. In: Interactive Models of Computation and Program Behaviour, Panoramas et Synthèses 27, Société Mathématique de France 1196 (2009)

[37] Rosebrugh, R., Wood, R.: Distributive laws and factorization. Journal of Pure and Applied Algebra 175(13), 327 - 353 (2002), special Volume celebrating the 70th birthday of Professor Max Kelly 
[38] Ross Duncan, K.D.: Interacting Frobenius algebras are Hopf. CoRR abs/1601.04964 (2016), http://arxiv.org/abs/1601.04964

[39] Selinger, P.: A survey of graphical languages for monoidal categories. Springer Lecture Notes in Physics 13(813), 289-355 (2011)

[40] Sleep, M.R., Plasmeijer, M.J., van Eekelen, M.C.J.D. (eds.): Term Graph Rewriting: Theory and Practice. John Wiley and Sons Ltd., Chichester, UK (1993)

[41] Sobociński, P., Stephens, O.: A programming language for spatial distribution of net systems. In: Petri Nets '14 (2014)

[42] Street, R.: The formal theory of monads. J Pure Appl Algebra 2(1), 243-265 (2002)

[43] Zanasi, F.: Interacting Hopf Algebras: the theory of linear systems. Ph.D. thesis, Ecole Normale Supérieure de Lyon (2015) 